\title{
Shock Waves in P-bar Target
}

\author{
Z. Tang and $\mathrm{K}$. Anderson \\ Fermi National Accelerator Laboratory \\ P.O. Box 500, Batavia, Illinois 60510
}

November 1991 


\section{Disclaimer}

This report was prepared as an account of work sponsored by an agency of the United States Government. Neither the United States Government nor any agency thereof, nor any of their employees, makes any warranty, express or implied, or assumes any legal liability or responsibility for the accuracy, completeness, or usefullness of any information, apparatus, product, or process disclosed, or represents that its use would not infringe privately owned rights. Reference herein to any specific commercial product, process, or service by trade name, trademark, manufacturer, or otherwise, does not necessarily constitute or imply its endorsement, recommendation, or favoring by the United States Government or any agency thereof. The views and opinions of authors expressed herein do not necessarily state or reflect those of the United States Government or any agency thereof. 


\section{Shock Waves in P-bar Target}

Zhijing Tang and Kris Anderson

Fermi National Accelerator Laboratory

April 15, 1991 


\section{Table of Contents}

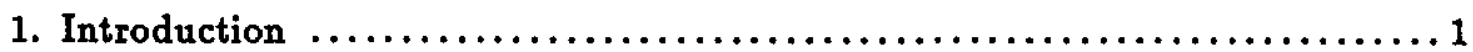

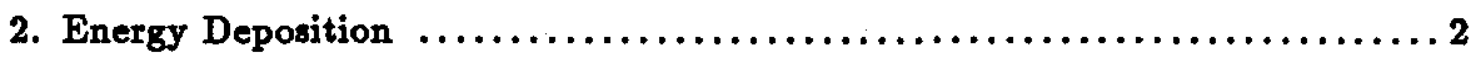

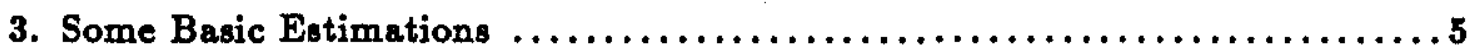

4. Waves Generated be Thermal Shock $\ldots \ldots \ldots \ldots \ldots \ldots \ldots \ldots \ldots \ldots \ldots . \ldots$

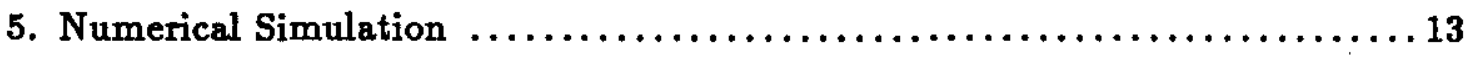

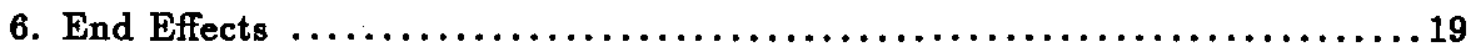

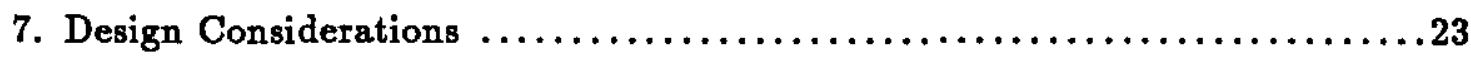

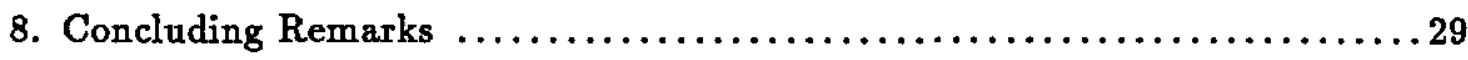




\section{Introduction}

The deposition of large amount of beam energy in short time will cause high temperature and pressure in the center of P-bar Target, and this disturbance will propagate outwards as a shock wave. Shock wave induced material changes which are of our concern include void growth and accompanying density decrease which will decrease antiproton yield, and crack formation and fracture as was observed in tungsten target which will destroy the integrity of the target.

Our objective is to analyze the shock wave behavior in the target, optimize its design so that the destructive effects of shock wave can be minimized, the integrity of the target can be maintained, and a reasonably high yield of antiproton production can be achieved.

In this report we put together some results of our analysis of a cylindrical copper target. We hope that it will provide a general overview of the shock wave phenomena in the target, establish a basis for further research, and facilitate the target design.

First, energy deposition data are analyzed, and it is justified that as an approximation, the problem can be treated as axi-symmetric. The averaged data therefore are used as energy profile, however, the maximum energy deposition are still used as the peak value. Next some basic estimations are made as to what temperature and pressure can reach at present level of energy deposition. Then some characteristics of wave propagation in a thermal shock loaded solid are illustrated with a one-dimensional model.

Since there is no analytical solution available for cylindrical geommetry, our understanding of the problem relies on numerical model, which are performed via finite element package ANSYS. results of numerical analysis are summarized, sources of potential danger are identified, and design ideas to minimize the damage are proposed. Finally, some restrictions to the results are pointed out. 


\section{Energy Deposition}

At present, about $1.7 \times 10^{12}$ protons per pulse at $120 \mathrm{GeV}$ are being used to produce p-bar from a $7 \mathrm{~cm}$ copper target, and about $1.0 \times 10^{7}$ p-bars per pulse are being stacked in the accumulator. Energy deposition data are provided by Bhat. Here the maximum energy density is 425 joules per gram, and the total energy is about 775 joules.

From the contour curves of the energy deposition data (see Fig. 1), we see that the most severe deposition is in a core region about $0.5 \mathrm{~mm}$ radius along the beam axis. Furthermore, the contour curves are almost parallel to the axile (note the difference in radial and axial scales). Hence, as a first approximation, we can assume that the problem is essentially two dimensional and axisymmetric. i.e. problem depends only on radial distance $r$ and time $t$.

Hence we average the data along the axis, and get the energy deposition as shown (Table I and Fig. 2 ). Notice that, in following analysis, the maximum energy density is still scaled at 425 joules per gram instead of 296 joules per gram. This represents a total energy deposition of 1113 joules.

Above averaged energy deposition data as a function of radial distance from target axile can be expressed by

$$
U= \begin{cases}U_{\text {max }} & r<r_{0} \\ U_{\text {max }} \exp \left\{-a\left(r-r_{0}\right)^{1 / 2}\right\} & r>r_{0}\end{cases}
$$

The constants $a$ and $r_{0}$ can be determined from the data

\begin{tabular}{|r|cccccccc|}
\hline element & 1 & 2 & 3 & 4 & 5 & 6 & 7 & 8 \\
$\mathrm{r}(\mathrm{mm})$ & .3125 & .9375 & 1.5625 & $\mathbf{2 . 1 8 7 5}$ & 2.8125 & 3.4375 & 4.0625 & 4.6875 \\
$\mathrm{U} / \mathrm{U}_{\text {max }}$ & 1.000 & $\mathbf{0 . 2 0 2}$ & $\mathbf{0 . 0 9 2}$ & 0.052 & 0.034 & 0.024 & 0.016 & 0.012 \\
\hline
\end{tabular}

We have

$$
a=2.114 \quad \text { and } \quad r_{0}=0.3125
$$

Equation ( 1 ) is also depicted in Fig. 2 as a dashed line. 


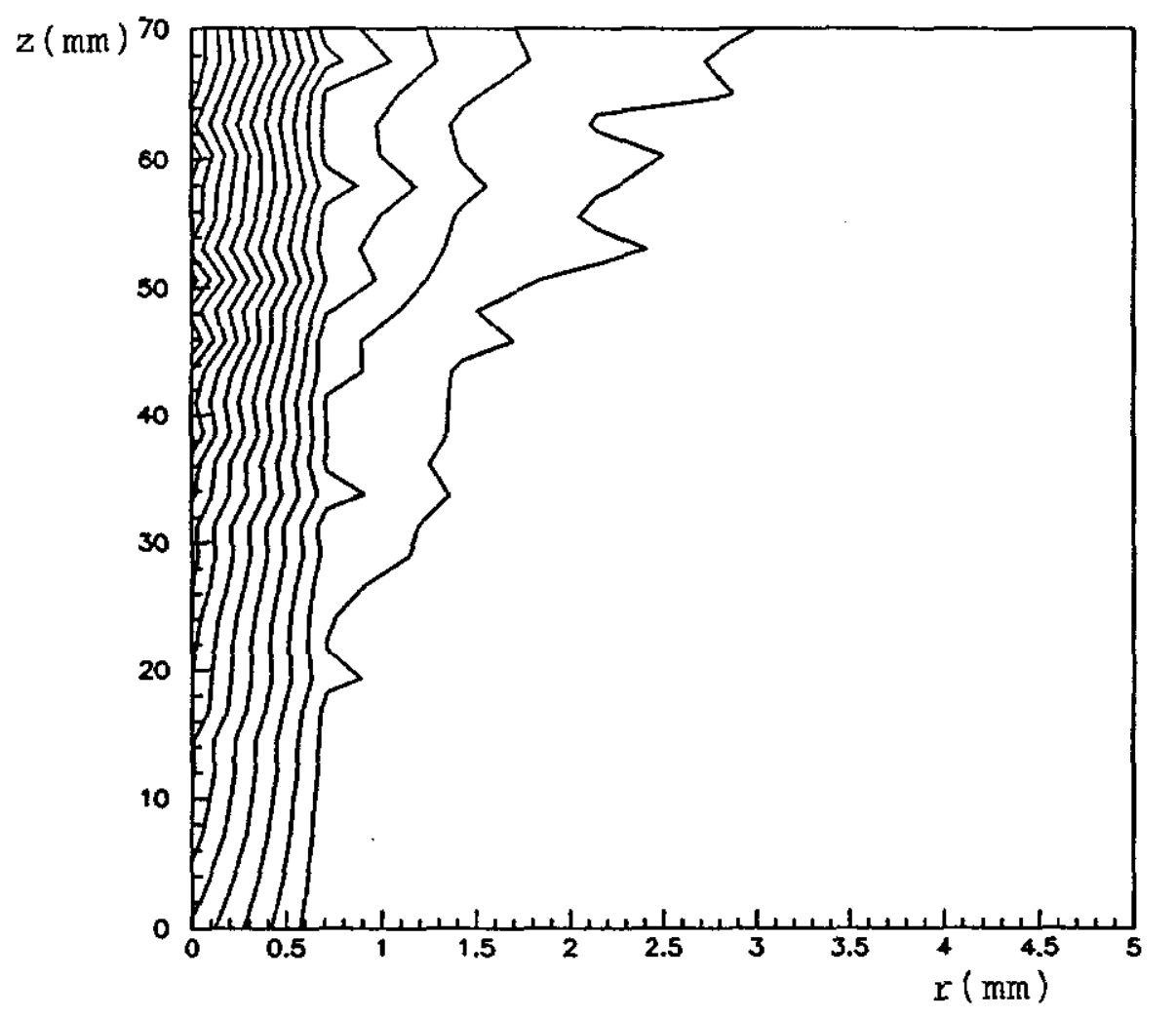

Fig. 1

contour curves of energy deposition

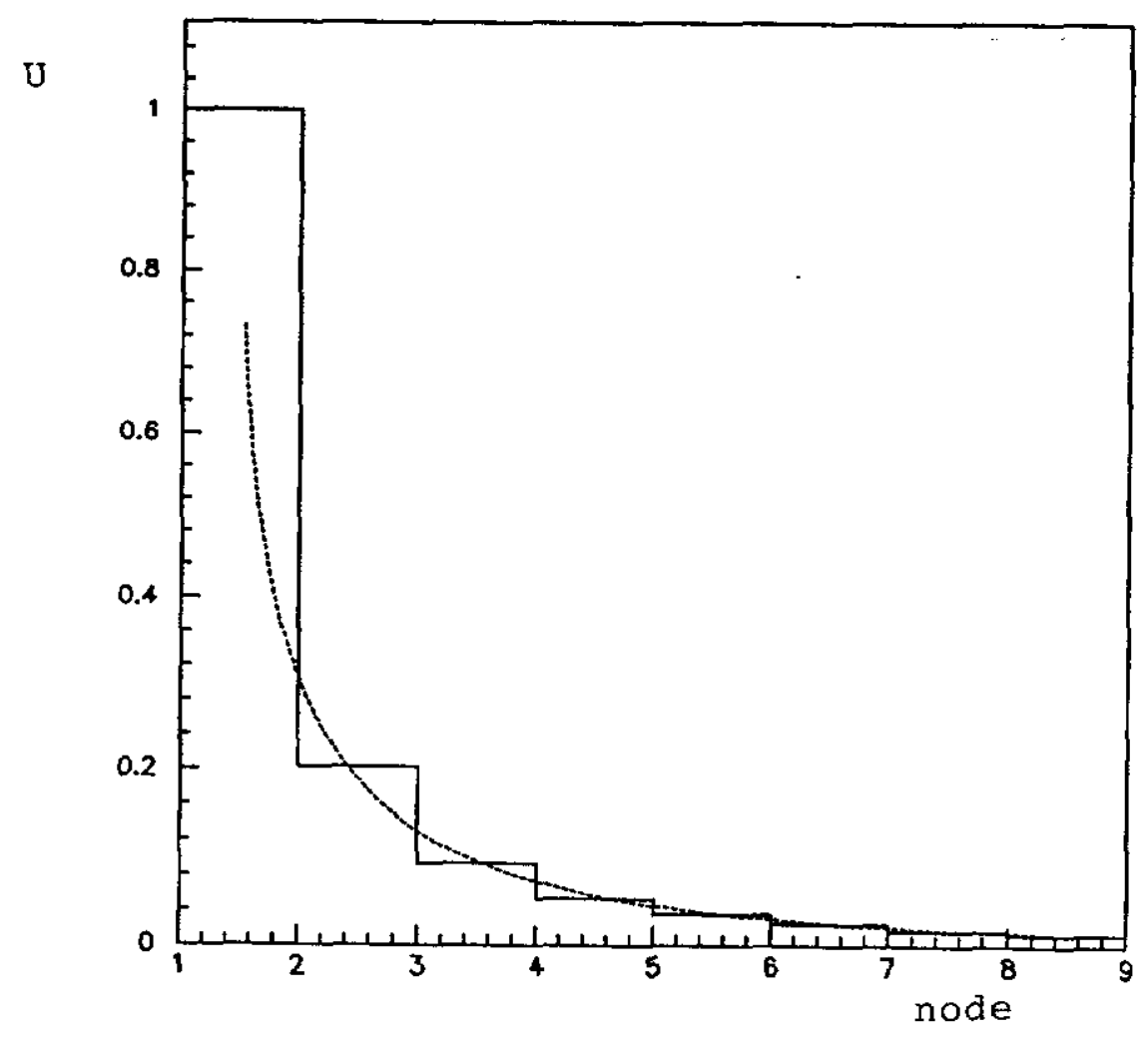

Fig. 2

Averaged energy profile 
Table I

Distribution of energy deposition

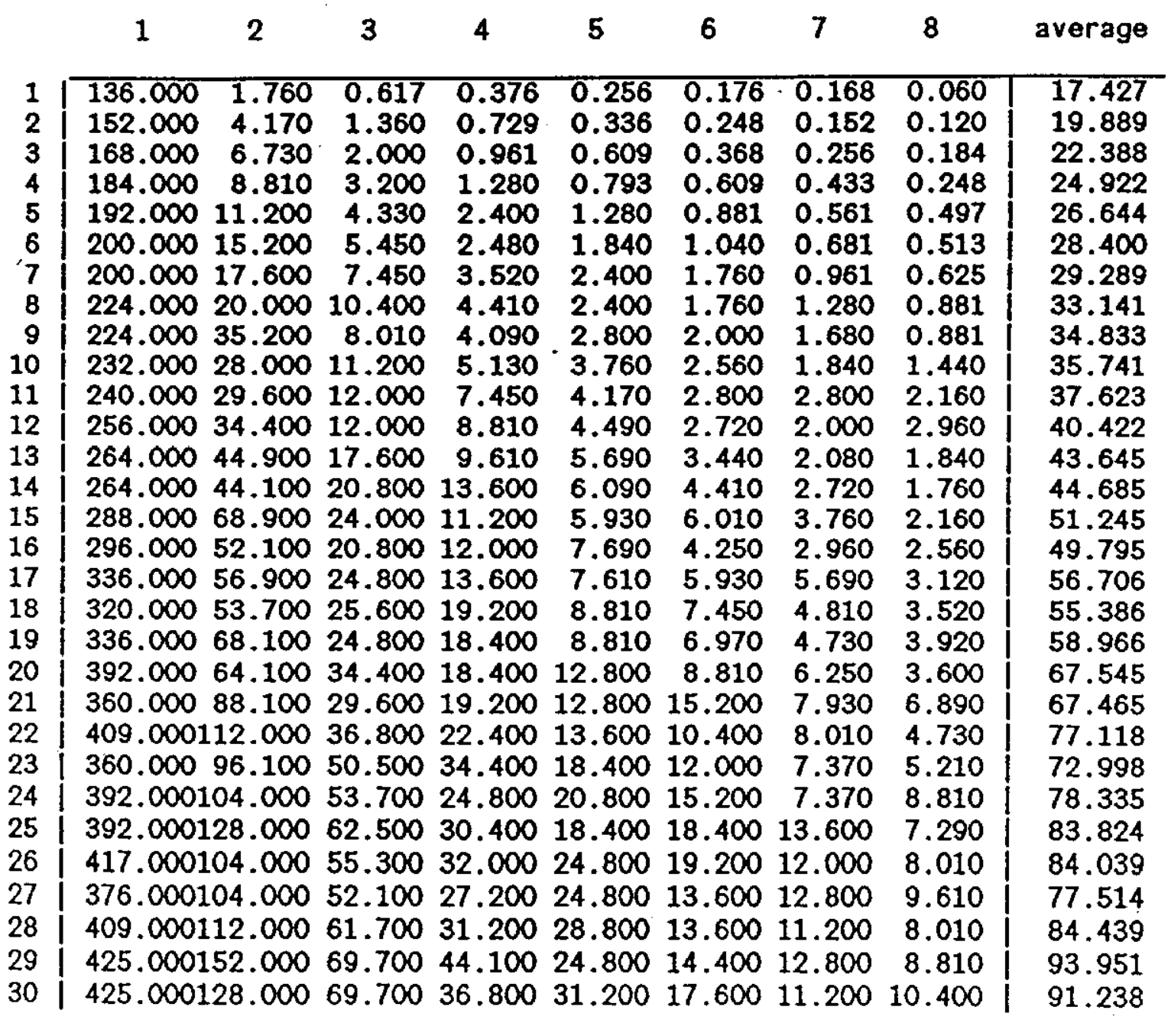

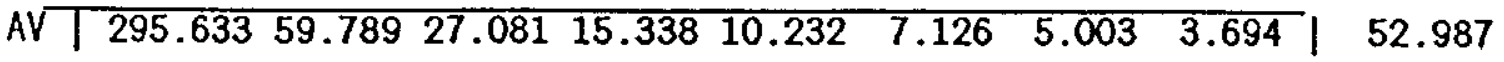




\section{Some Basic Estimations}

When large amount of proton beam energy is deposited in the target, temperature increase, so does pressure. Since the energy deposition is inhomogeneous, it causes mechanical nonequilibrium as well as thermal nonequilibrium, hence results in material motion and heat conduction. Because the speed of wave propagation and that of heat conduction are different, as long as the energy deposition is not too large, such that no strong shock is developed, these two kinds of problems can be dealt with separately.

In following, we will give some basic estimations for our problem: How high the temperature can reach in the target; what is the corresponding pressure; How is the energy be divided into mechanical energy and thermal energy, and what is their approximate ratio. Some of the results will be used in numerical analysis, some are used just for facilitating the understanding of the problem and double check purpose.

Because the length of the proton beam pulse is so short, there is no time for any significant transportation process, either mechanical or thermal, to take place during energy deposition. Therefore the process can be adequately described as an adiabatic and equivolumitric heating. Hence

$$
U=\int_{\theta_{0}}^{\theta_{1}} c_{v} d \theta
$$

where $U$ is energy deposition, $c_{v}$ is specific heat at constant volume, and $\theta$ is the absolute temperature.

For solid materials, handbooks give only the specific heat at constant pressure, namely $c_{p}$, we have to find an approximation for $c_{v}$. From thermodynamics relation and definitions for $c_{v}$ and $c_{p}$, we can get

$$
c_{p}-c_{v}=V \alpha_{v}^{2} \theta / \beta
$$

where $V$ is the specific volume of the solid, $\alpha_{v}$ is the volume thermal expansion coefficient, and $\beta$ is the compressibility of the solid.

From the Mie-Grüneisen equation of state for solids, we have

$$
\gamma=V \alpha_{v} / \beta c_{v}
$$

Combine above two equations, we can write 


$$
c_{p} / c_{v}=1+\gamma \alpha_{v} \theta
$$

For copper, $\gamma=1.96, \alpha_{v}=49 \times 10^{-6}\left({ }^{\circ} \mathrm{K}\right)^{-1}$, above equation gives

$$
c_{p} / c_{v}=1+96.5 \times 10^{-6} \theta=1.026+96.5 \times 10^{-6} \mathrm{~T}
$$

where $T$ is the temperature in centigrade. Note that, when $T=0$, the difference between the two specific heat is only $2.6 \%$, and when $T=1000^{\circ} \mathrm{C}$ the difference is $12.3 \%$.

From handbooks, the specific heat at constant pressure of copper in the temperature range between room temperature and melting point can be approximated as

$$
c_{p}=0.385(1+0.000304 T) \text { Joules } / \mathrm{g}^{-}{ }^{\circ} \mathrm{C}
$$

Therefore, $c_{v}$ can be approximated by

$$
c_{v}=0.375(1+0.0002 T) \mathrm{Joules} / \mathrm{g}^{\circ} \mathrm{C}
$$

Substitute into equation (3), we get energy deposition. rearrange we have an equation for temperature $T$

$$
T+0.0001 T^{2}=1133+T_{0}+0.0001 T_{0}^{2}
$$

Let $T_{0}=25$, above equation gives $T=1052^{\circ} \mathrm{C}$, below the melting temperature of copper $\left(T_{m}=1083^{\circ} \mathrm{C}\right)$.

Next we will estimate the pressure caused by energy deposition. From Grüneisen equation

$$
P=\rho \gamma U=7.4 \mathrm{GPa}
$$

where $\rho$ is the mass density, $\rho=8.94 \mathrm{~g} / \mathrm{cm}^{3}$. From thermoelasticity, we get

$$
P=\frac{E}{1-2 \nu} \alpha\left(T-T_{0}\right)=6.9 \mathrm{GPa}
$$


where $E=123 \mathrm{GPa}$ is the Young's modulus, $\nu=0.35$ is Poisson's ratio, $\alpha=1.64 \times$ $10^{-5}\left({ }^{\circ} \mathrm{C}\right)^{-1}$ is the linear thermal expansion coefficient.

In (11) pressure in proportional to energy deposition whereas in (12) pressure is proportional to temperature increase, and by (10) temperature increase is not a linear function of energy deposition, but the difference is small. Hence as a first approximation, we can use thermoelasticity to calculate pressure. From these two independent results, we know the pressure can reach as high as $7 \mathrm{GPa}$ at the center of the target.

As previously mentioned, the energy deposition is inhomogeneous through out the target with most of the energy deposited in the core. Therefore the state right after the energy deposition is highly unstable, the region with higher pressure tends to expand into its surrounding region, set the material mass into motion, and hence convert part of the energy into mechanical energy. Assume this process to be quasistatic, the energy to be converted can be estimated as

$$
\bar{U}_{M}=\int P d V=P^{2} / 2 \kappa=179 \text { Joules } / \mathrm{cm}^{3}
$$

where $\kappa=E / 3(1-2 \nu)=137 \mathrm{GPa}$ is the bulk modulus, and we take $P=7 \mathrm{GPa}$. Since the density $\rho=8.94 \mathrm{~g} / \mathrm{cm}^{3}$, we have

$$
U_{M}=20 \mathrm{Joules} / \mathrm{gram}, \text { and } U_{M} / U=4.7 \%
$$

That is, only about five percent of the total deposited energy is converted into mechanical energy, which is responsible for wave motion.

Note that, above estimate implies two simplifications. First we replace the inhomogeneous deposition of energy with a homogeneous one. That is there is no internal constraint other than inertia, and at the end of the process the pressure is completely released. In fact, there is always a thermal-elastic equilibrium pressure as the result of inhomogeneous heating. A thermoelastic analysis is performed and the result is $P_{\text {equilibrium }}=3.7 \mathrm{GPa}$. This gives a factor of 0.72 to above approximation. Second, we assume the pressure release process to be quasi-static. The real process is strongly dynamic, and this will often result in an overshot above the final equilibrium state. A factor of 1.5 is adequate to correct the result. These two factors tend to cancel each other, and as a rough estimate, above result is good enough.

Another phenomenon of interest is the melting of target material. In Fig. 3 we depict temperature $T\left(\times 1000^{\circ} \mathrm{C}\right)$ as a function of energy deposition $U$ (Joules/gram). Also depicted in the figure is the melting temperature, which increases with pressure 
(about $35^{\circ} \mathrm{C} / \mathrm{GPa}$ ). From the figure we see that, when energy deposition reaches 600 Joules/gram, the temperature reaches melting point. The heat needed to melt copper is about 200 Joules/gram, therefore, there will be no melting when energy deposition is below 800 joules/gram.

Now sum up main conclusions of this section: As the result of proton beam energy deposition, the enthalpy of target material increases, part of which in the form of internal energy, hence increase temperature, and part of which in the form of potential energy, hence increases pressure. The later part will eventually be released as the mechanical energy of stress waves. For a maximum energy deposition of 425 Joules/gram, temperature can reach $1052^{\circ} \mathrm{C}$, pressure can reach $7 \mathrm{GPa}$, and only $5 \%$ of the total energy is converted into mechanical energy. It should be noted that, although the mechanical energy is small compared with thermal energy, it is more difficult to be taken out of the target than the thermal energy, and must be dissipated in some way.

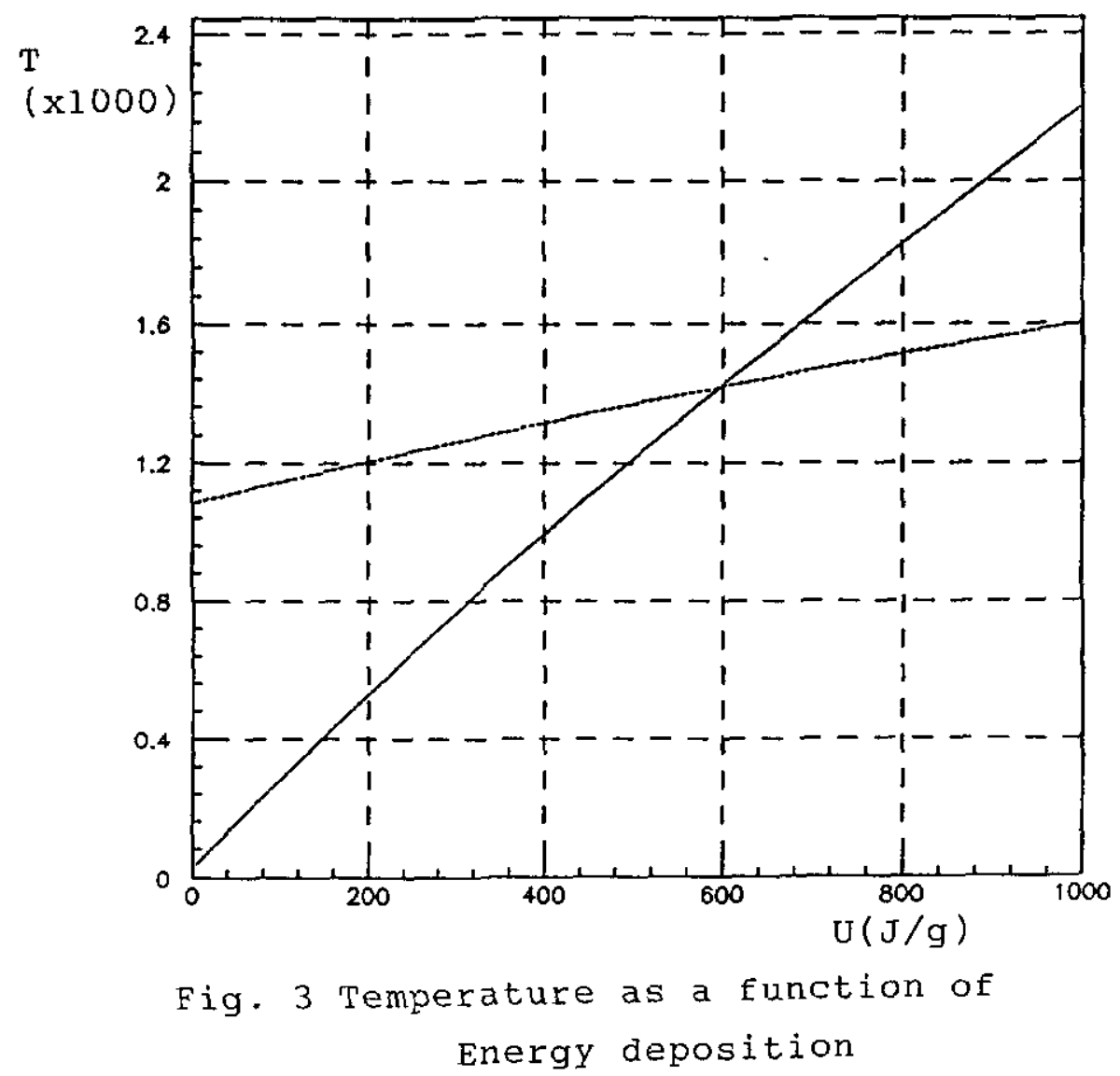




\section{Waves Generated by Thermal Shock}

The sudden deposition of energy will cause stress waves to propagate in the P-bar target. These waves includes elastic waves, plastic waves, and even shock waves. In the center of the target the pressure well exceeds the yield stress of the target material by an order of magnitude, this will result in shock wave propagation; as the waves propagate outward, energy spreads out, pressure drops down, the waves decay into elastic- plastic waves; as the waves propagate further, they will eventually become elastic waves.

The detailed study of these waves can be quite involved, and even intractable, since different models describing different wave phenomena must be incorporated to describe the whole wave propagation process, and numerical techniques are indispensable to get final result such as stress, strain and particle velocity distribution both in time and space. Nevertheless, some basic characteristics of stress wave propagation in the target can be described by elementary wave theory.

In this chapter we use simple wave theory to study the characteristics of waves produced by thermal shock loading. How the amplitude of wave changes as it propagates through the target; How the reflection takes place at free boundary and at the center of target; What causes potential danger to our target.

Assume that the energy deposition is accomplished instantaneously at time $t=0$, and the result of the initial energy deposition is the adiabatic heating in the core of a circular cylinder to a temperature $T_{0}$ referred to the ambient temperature. We further assume that the material response is purely elastic and the elastic constants does not depends on temperature. We also assume that the time scale for heat transfer is much larger than that for wave propagation, hence temperature does not change during wave propagation process.

Above problem is equivalent to one in which the core region of a circular cylinder is initially under a pressure $P_{0}$, the boundary condition at out surface is free whereas the boundary condition at center is fixed by symmetry. The high pressure region will expand to its surrounding, thus causes wave motion. Due to mathematical difficulties of cylindrical wave, only wave front solutions can be obtained analytically. Thus, for illustrative purpose, we consider a one-dimensional problem: an elastic bar, left end is fixed, right end is free, initially at rest, and a portion of the bar at left end is initially under pressure (Fig. 4), find the motion and stress history.

The solution of above problem is depicted in Fig. 5,6 and 7. Fig. 5 is the $P-V$ loci of the solution; Fig. 6 is the $X-t$ plane where we can see how wave propagates back and forth between two boundaries; and Fig. 7 are some snapshots of solution 
at different times. From the figures, we see that the amplitude of pressure wave is half the initial pressure whereas the length of the wave is twice of initial length of the pressured region. When wave arrives at free boundary, pressure becomes zero while velocity doubled. Upon reflection, the compressive wave becomes tensile wave. When this tensile wave arrives at fixed boundary, velocity becomes zero while stress doubled.

Same kind of phenomena happen in our circular cylinder target. Compressive wave propagates outward, and is reflected back from free surface as a tensile wave. It is the implosion of this tensile wave which is potential danger to our target and should be minimized.

The evolution of wave amplitude can be obtained by a simple energy flow consideration. The energy flux of a stress wave is proportional to the square of stress, and for a circular symmetric wave the area of wave front is proportional to the radius. In order the energy to be conserved, the product of the square of stress and the radius must be constant. That is, the stress is inversely proportional to the square root of the radius. Thus, for an outward going wave, amplitude decreases as wave propagates. But for an inward going wave, the amplitude increases as wave propagates. In our problem, as the wave reflected from free surface propagates into the core of the cylinder, the tensile stress become large and large. Therefore, the most dangerous region is the core of the cylinder.

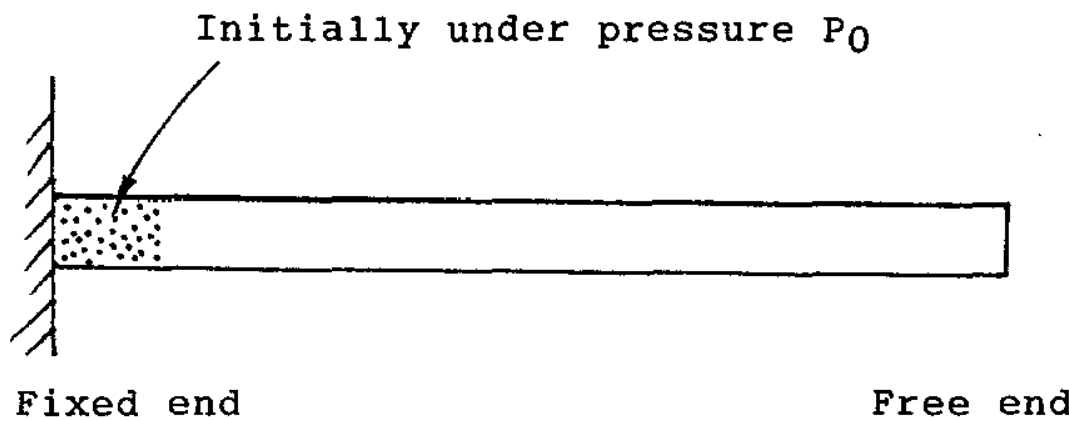

Fig. 4 A one-dimensional model 


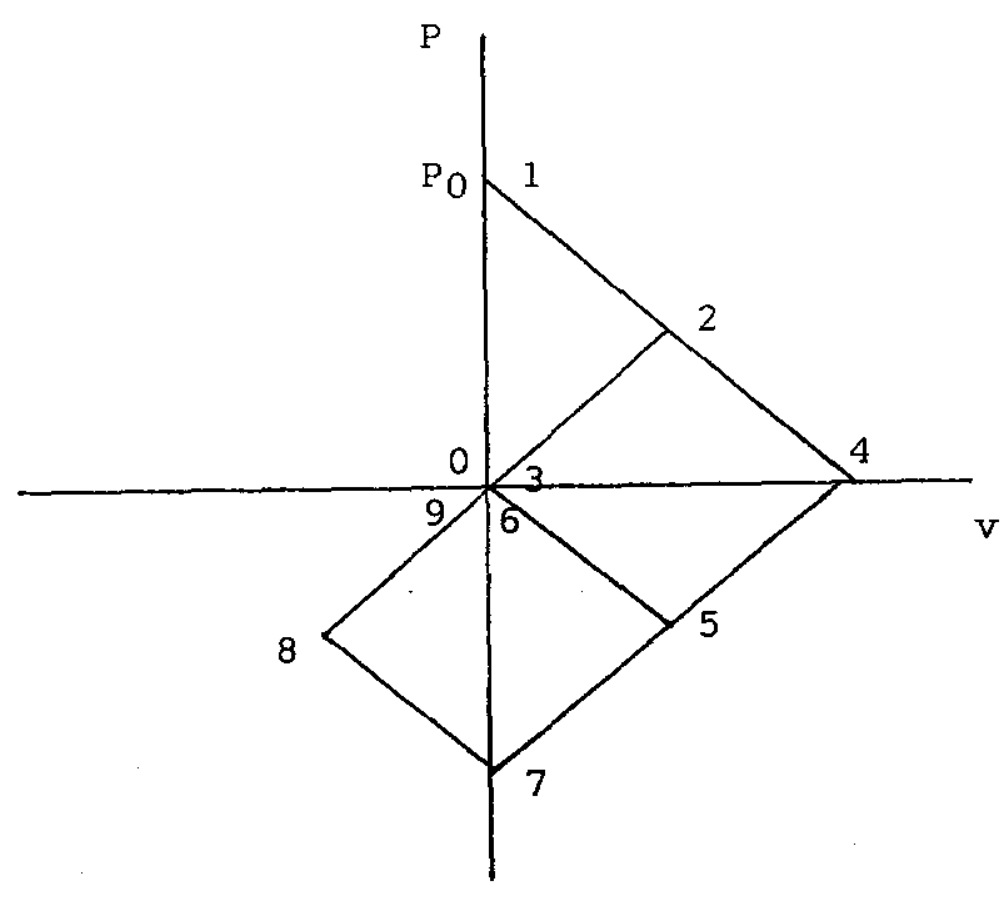

Fig. 5

$t$

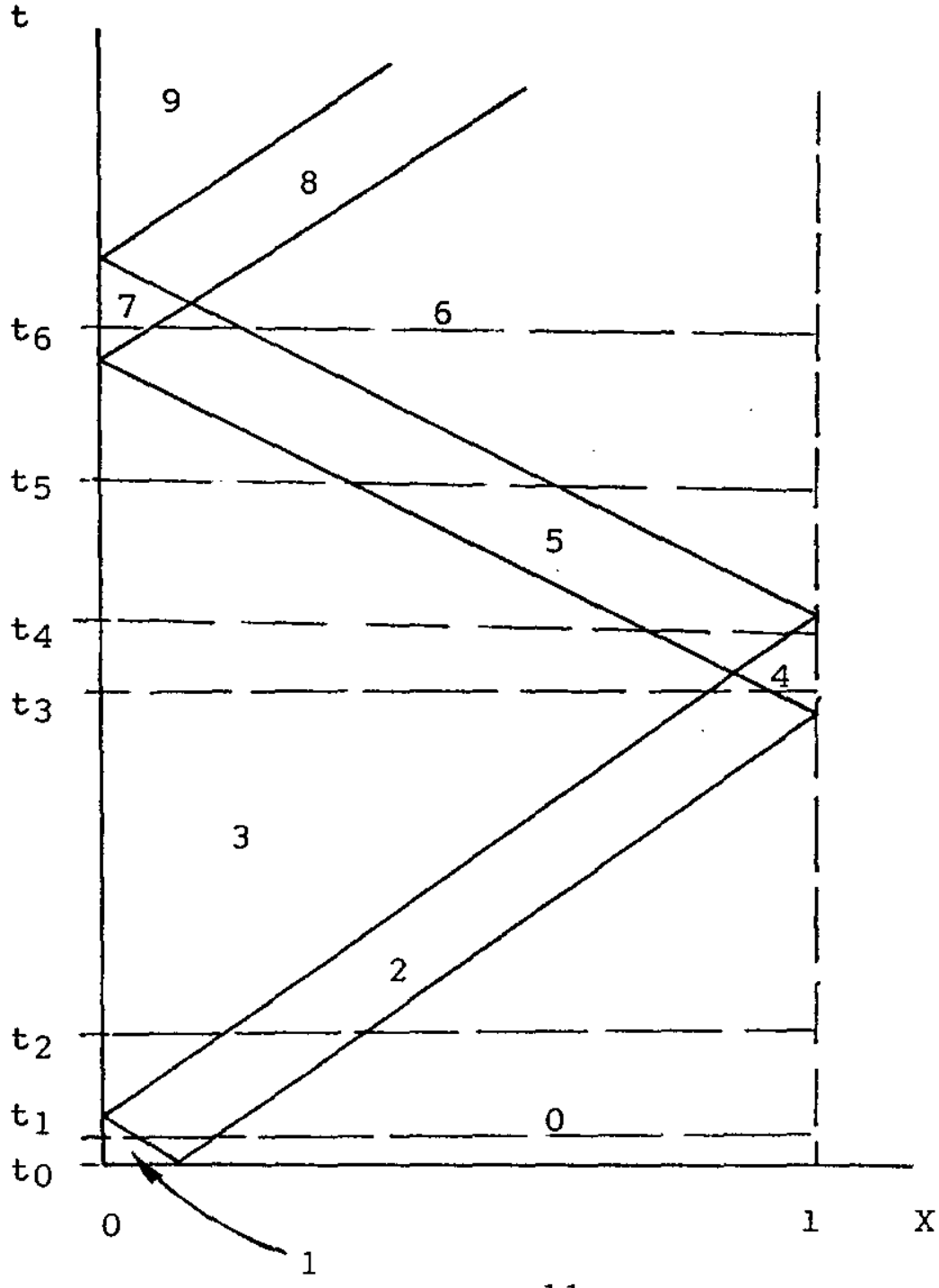

11

Fig. 6 


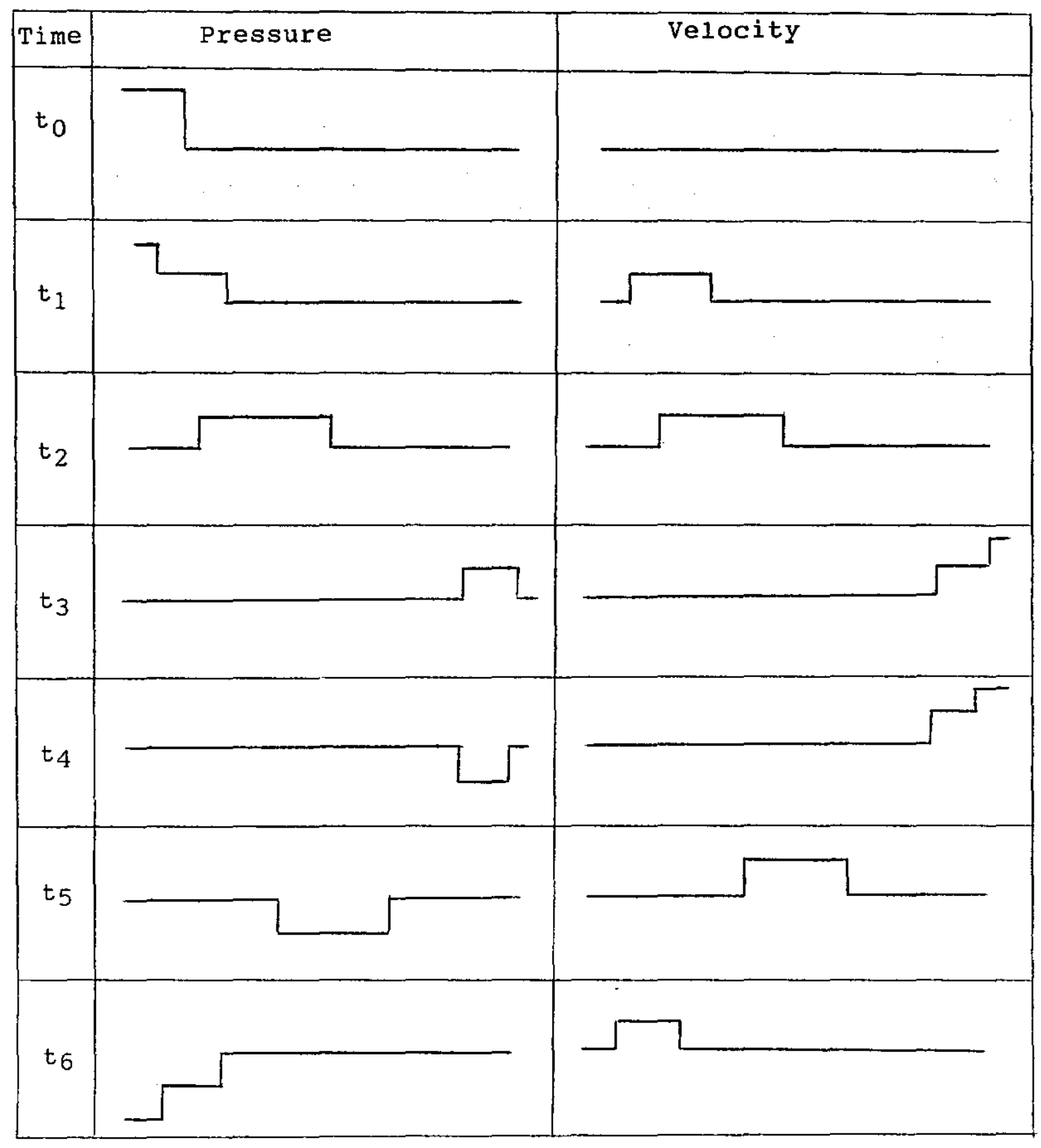

Fig. 7 solutions at different times 


\section{Numerical Simulation}

In this chapter, the results of numerical simulation will be summarized.

The finite element program package ANSYS is used to investigate the stress wave propagation phenomena in P-BAR target for an idealized case, in which the energy deposition is assumed to be accomplished as the result of the adiabatic heating of a circular cylinder. This temperature rise will then produce high pressure due to the thermal coefficient of linear expansion of the material. And this high pressure will set the cylinder into wave motion. The material response is assumed to be elastic-plastic which is governed by von Mises yield criteria with kinematic hardening. The stress strain curve is temperature dependent. We also assume that the time scale for heat transfer is much larger than that for wave propagation, hence heat transfer does not come into the picture during wave propagation process.

Fig. 8 is a 3-D visualization of the wave pattern which plots displacement as a function of time and radial distance. Fig. 9 is the projection of Fig. 8 onto timedisplacement plane. Here we see clearly how a compressive wave propagates outwards and be reflected back at the free boundary. The reflected wave is a tensile wave which propagates inwards the center of the cylinder. Since the zero displacement at the cylinder center, the center is equivalent to a rigid boundary, when the in-going tensile wave reaches center, it is reflected back as a tensile wave.

We can also see how the amplitude of wave decreases as it propagates outwards, and increases as it propagates inwards. The evolution of amplitude for both outward going wave and inward going wave are depicted in Fig. 10, and comparison with the inverse square root law is satisfactory.

Fig. 11 and 12 give radial and hoop stresses in first five center elements. The variation of stresses across the nodes are quite monotonic. After initial compressive wave, the radial stress is in compression, while the hoop stress is in compression only until element 3 , beyond which tension prevails in hoop direction. The maximum tensile stresses occur in both radial and hoop directions in element 5 at time around $t=12 \mu s$, this is caused by the tensile wave reflected from free boundary. Damage processes such as void growth and fracture can occur only under tension. From our analysis, however, the tensile stress is not enough to cause any above mentioned damages. Another point we want to emphasis is that the stress in element 1 is basically isotropic which causes material expansion and the maximum density change can reach $5 \%$. The stress state in element 2 through 5 is under severe shear with maximum shear stress in element 2 and 3 , which may also cause some damage.

Fig.13 and 14 give plastic strains in radial and hoop directions, respectively. Note 
that the radial and hoop plastic strains in element 1 are both positive, this is consistent with our previous stress observation. Radial and hoop strains in other elements are of opposite sign, which means the deformation in these elements are basically shear. excessive shear deformation will definitely cause damage to target material.

The general picture of finite element analysis results can be abstracted as follows:

The stresses can be decomposed into two parts, static part and dynamic part. The static part is that of thermal stresses due to inhomogeneous temperature distribution, and the dynamic part is due to wave propagation effect.

For thermal stresse, the central part of the target is at high tempreture, hence it tends to expand itself; the neighboring part is at low temperature and acts as a confining vessel. Thus the stresses for central part is almost an isotropic pressure, and the stresses for the rest of the target is that of a thick cylindrical vessel under internal pressure.

For dynamic stresses, shcok wave begins at the center of the cylinder as a compressive pulse and propagates outwards, and following it there is a tensile pulse or rerafaction wave. Upon arriving at the free boundary, the compressive wave is reflected as a tensile wave, and the tensile wave becomes compressive. As this reflected tensile wave propagates towards center of the target, its amplitude increases. It is this tensile wave which may become dangerious to the target.

Add above two componants, we get overall stresses in the target. At the very center, the stress state is an isotropic pressure, that means the radial stress and the hoop stress are almost of the same value, and are both negative. This pressure is so high, that even when the reflected tensile wave cannot get it out of pressure. As one move outwards, both the radial stress and hoop stress decrease in magnitude, but the hoop stress decreases much more quickly than the radial stress, that means the stress state is no more isotropic, and large plastic deformation is expected. Further away from the center, the hoop stress becomes tensile. It is in this region, we have maximum tensile stress when wave reflected from free boundary arrives. Further more, the radial stress can become tensile. Therefore, this is the most dangerious region of target.

Let's summarize our main conclusions for this chapter: The characteristics of wave propagation in a circular cylinder target is investigated numerically, the results agree with the conclusion from previous chapter. The most significant density change occurs in element 1 ; The maximum plastic shear deformation occurs in element 2 and 3; The maximum tensile stress occurs in element 5. And the most important conclusion we can state is that, under present level of energy deposition, the circular cylinder copper target is safe. 


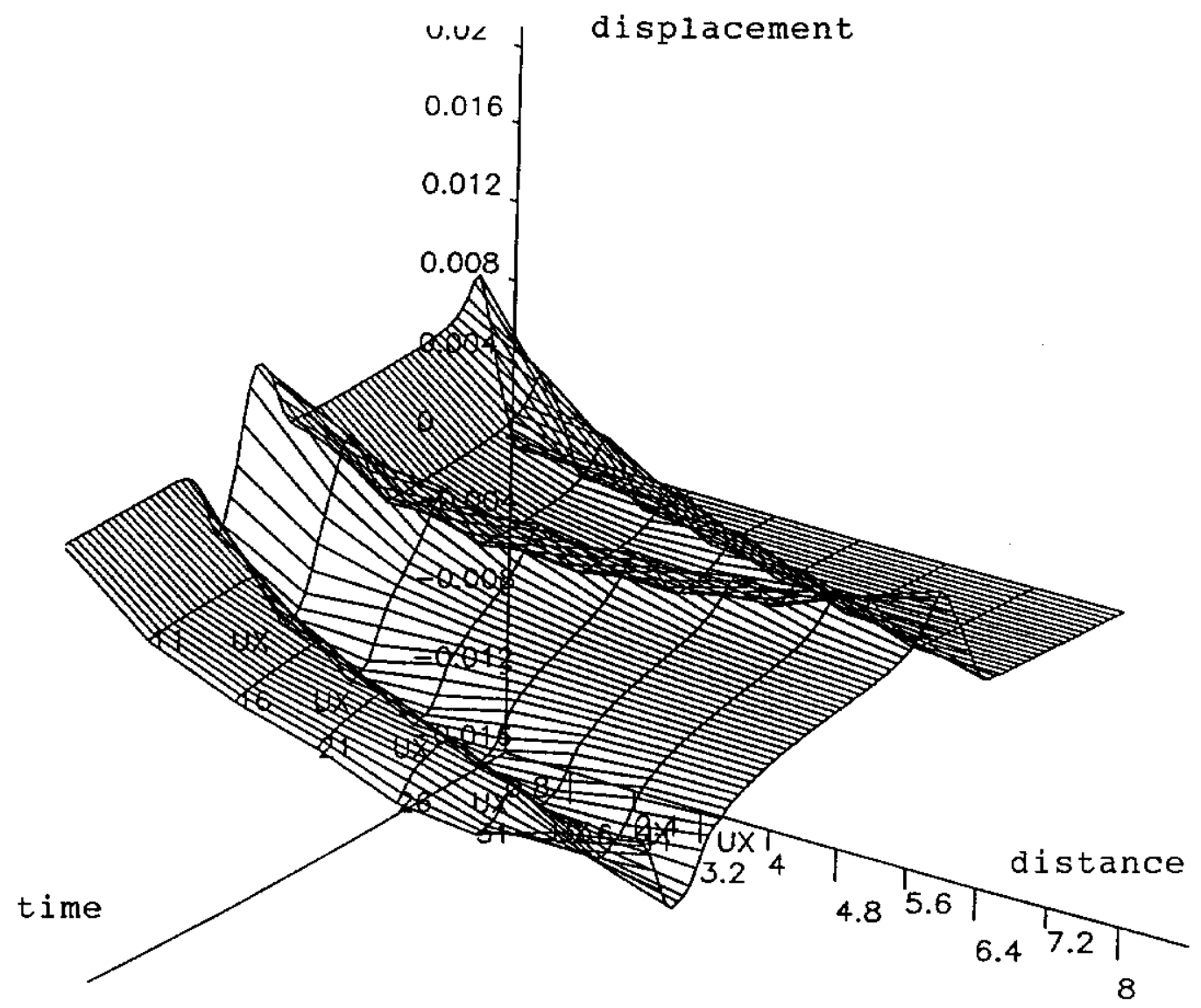

Fig. 8 Displacement as a function of distance and time. 

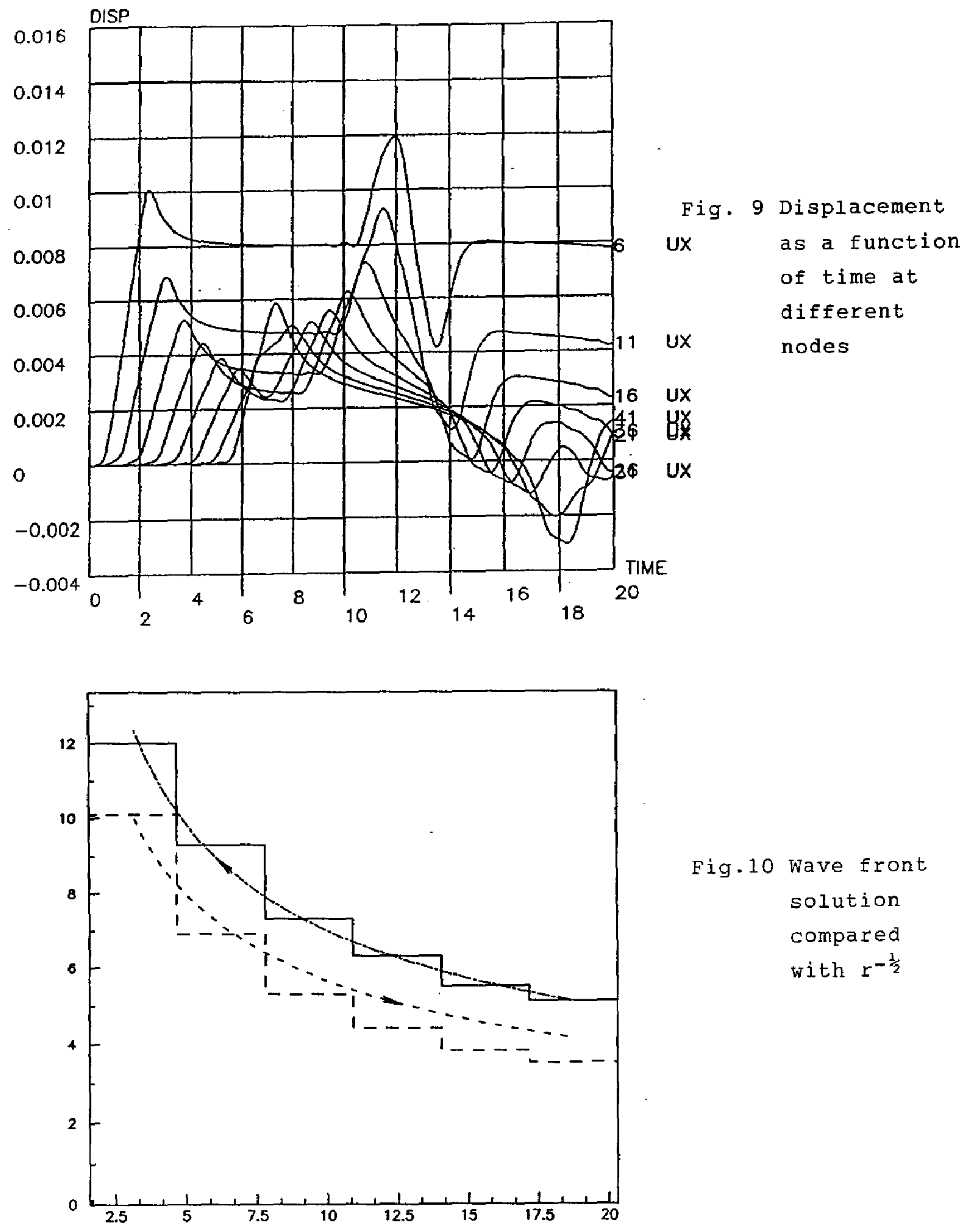

Fig. 10 Wave front solution compared with $r^{-\frac{1}{2}}$ 


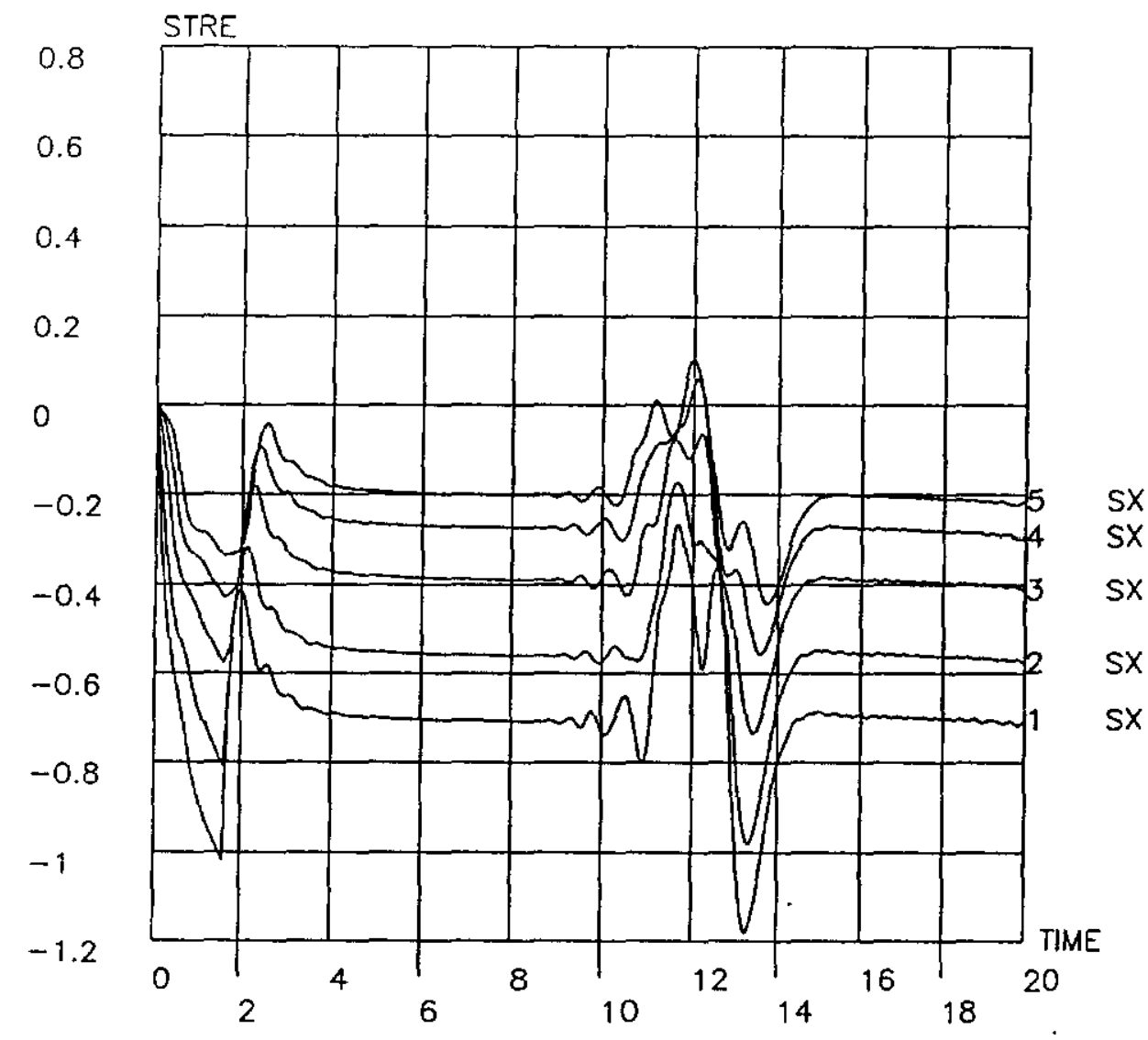

Fig. 11 Radial stress

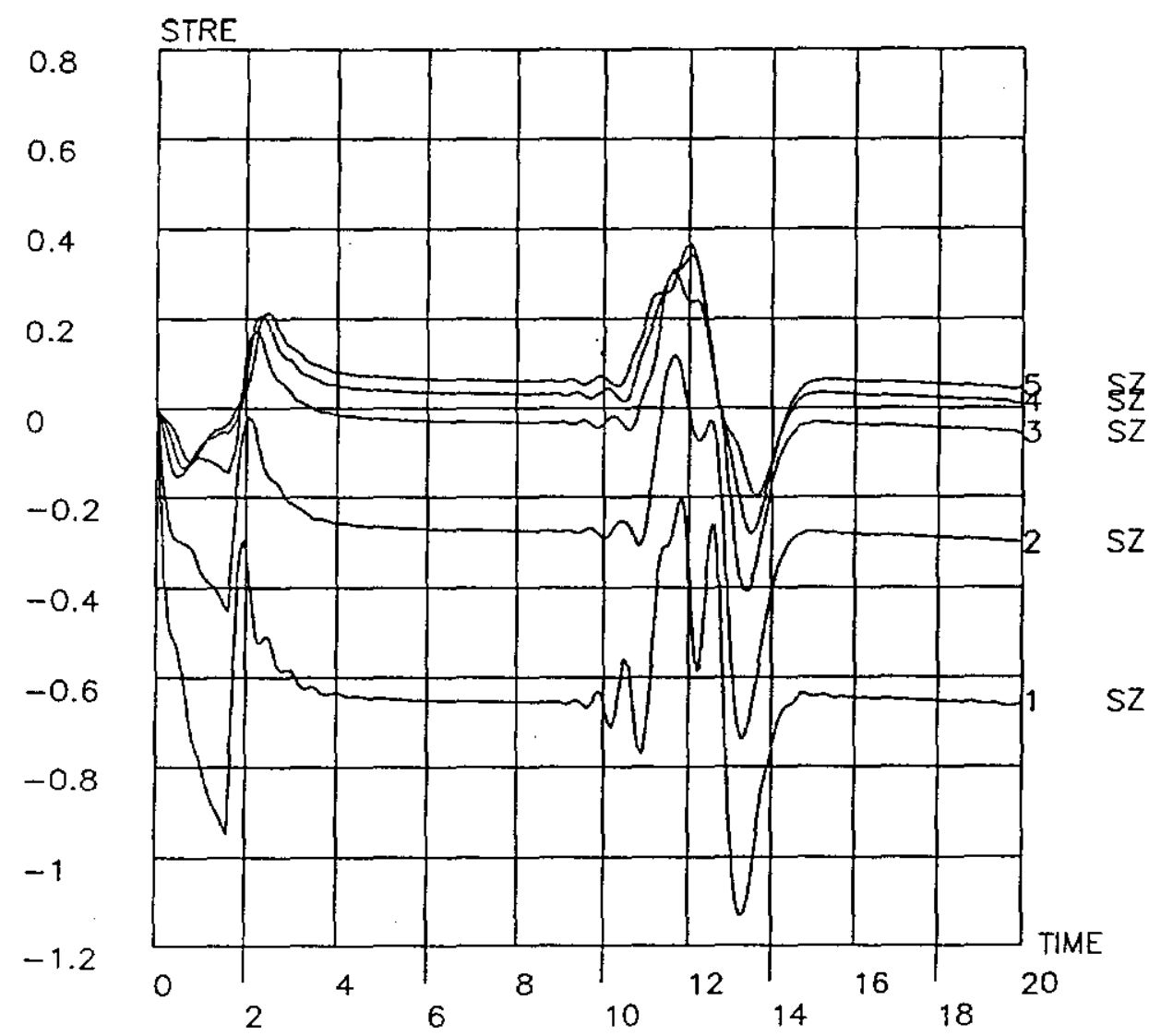

Fig. 12 Hoop stress

$Z$

$S Z$ $S Z$ 


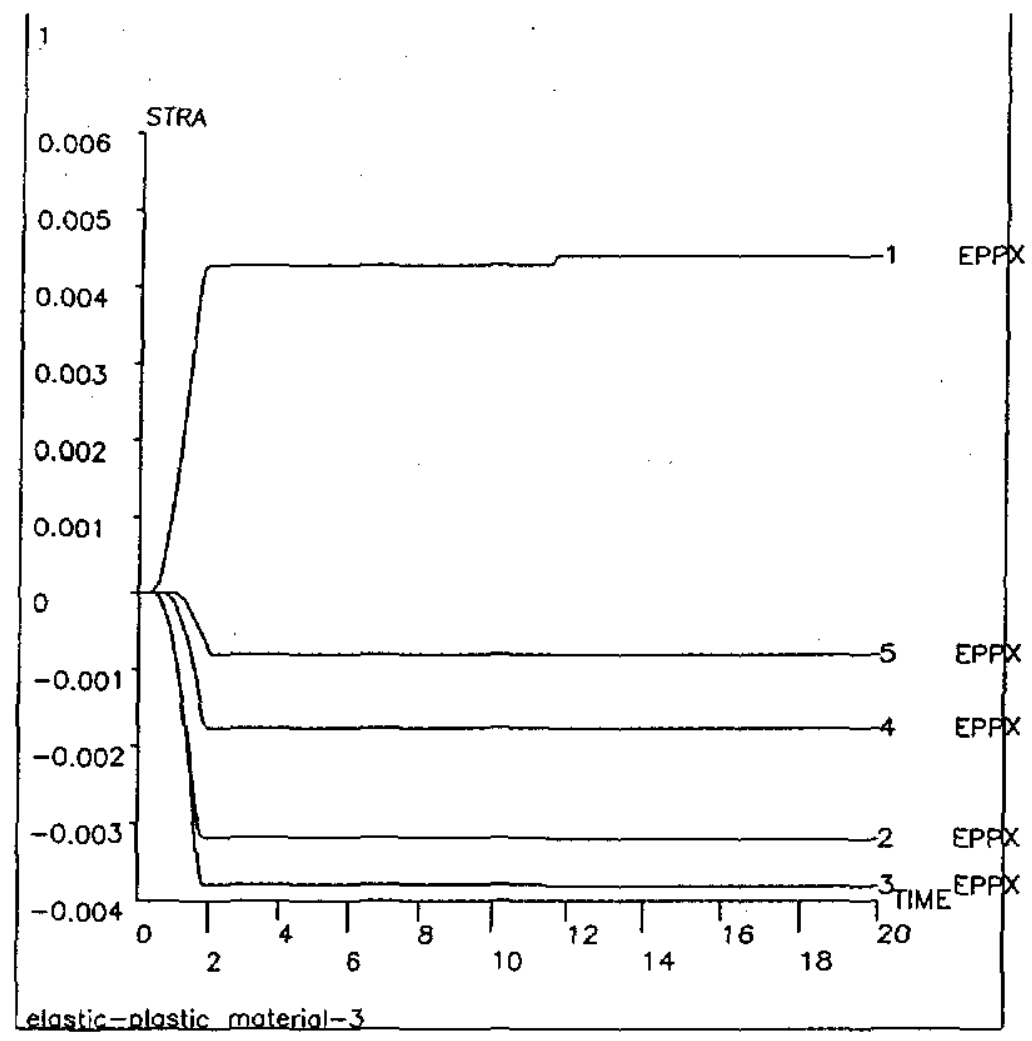

Fig. 13 Plastic strain in radial direction

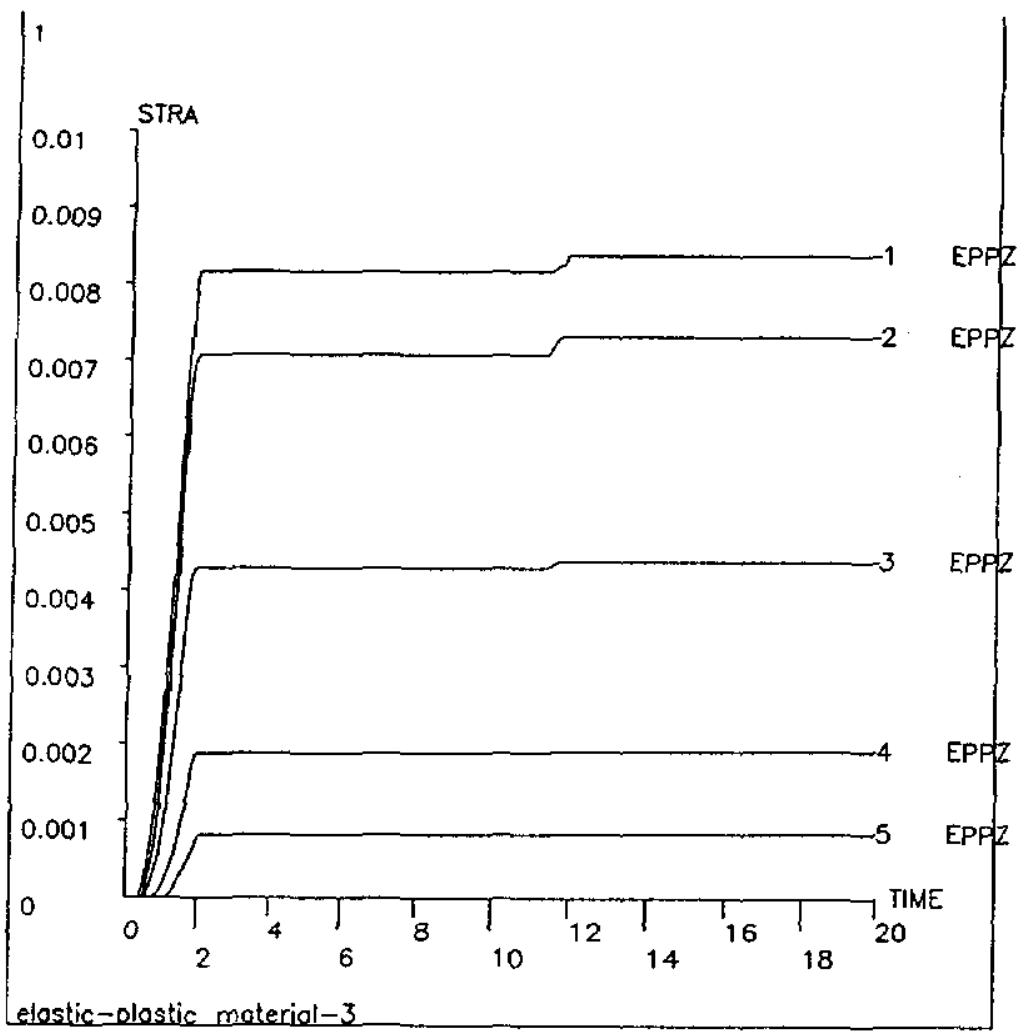

Fig. 14 Plastic strain in circumferencial direction 


\section{End Effects}

In previous studies we basically used one-dimensional model, that is assume the motion to be axially symmetric and there in only radial displacement. However, when we consider a finite target, above assumptions no longer apply. Although the axial symmetry still hold, there will be considerable axial displacement near the ends.

To check the end effects, we ran a two-dimensional model using ANSYS. We consider a circular cylinder of 5 centimeter diameter and 7 centimeter length. The specimen is divided into 10 elements along its radial direction and 30 elements along its axial direction. The material is assume to thermoelastic.

Other assumptions are the same as in previous studies except that there is no constraints on axial deformation. The initial energy deposition is realized by heat input during the first $1.6 \mu \mathrm{s}$ in two central layers of elements.

The material properties inputed into the program are

\begin{tabular}{|r|r|l|}
\hline quantity & value & unit \\
\hline mass density & 8.940 & $\mathrm{~g} / \mathrm{cm}^{3}$ \\
Young's modulus & 123.5 & $\mathrm{GPa}$ \\
Poisson's ratio & 0.350 & $\mathrm{NA}$ \\
coefficient of thermal expansion & 16.40 & $10^{-6} /{ }^{\circ} \mathrm{C}$ \\
\hline
\end{tabular}

The main results are presented in two figures.

Fig. 15 depicted deformed structure at different time together with undeformed structure of the target.

At $t=0$, the structure is undeformed. At $t=1.6 \mu \mathrm{s}$, just the end of energy deposition, the deformation is confined in first four layers of elements. As time goes on, the deformation propagates outwards. And at time $t=4.8 \mu \mathrm{s}$, it reaches the outer boundary. After that, a tensile wave is reflected back towards center. From the figures we see that this tensile wave reaches the center elements at about time $t=9.6 \mu \mathrm{s}$ when the center elements have maximum radial expansion.

Besides this main disturbance, we can also see the end effects from the figures. At time $t=1.6 \mu \mathrm{s}$, the elements at upper left corner have very large distortion as well as rotation. This is because they are pushed unbalancedly from inside. As time goes on, these distortion and rotation also propagate both into the target and along the boundary. In fact we can very clearly see the disturbance propagating first on the 
upper end surface outwards, then down the outer surface of the cylinder. It is clear that after time $t=11.2 \mu s$, the deformation everywhere in the cylinder is basically three dimensional.

However, from the figures it is also clear that there is no overall axial deformation except in upper left corner elements. The disturbances from ends are small compared with the radial disturbance. Hence if we are not particularly concerned about the upper left corner of the target, the one-dimensional model can still be used to examine the basic events resulted from sudden energy deposition.

The upper left corner of the cylinder corresponds to the center of down the beam end of the target, where energy density is maximum. From our analysis, there the distortion is largest and most non-uniform. It is this portion of the target where damage may develop first. Special precaution may be necessary to prevent the failure.

Deformation in the figures are extremely exaggerated to assist the visual examination. The maximum nodal displacement is less than $0.06 \mathrm{~mm}$. With mesh size of $2.5 \mathrm{~mm}$, this corresponds to a maximum strain of 0.024 .

Fig. 16 is some contour lines for equivalent stress, which is defined as

$$
\bar{\sigma}=\frac{1}{\sqrt{2}}\left\{\left(\sigma_{1}-\sigma_{2}\right)^{2}+\left(\sigma_{2}-\sigma_{3}\right)^{2}+\left(\sigma_{3}-\sigma_{1}\right)^{2}\right\}^{1 / 2}
$$

Equivalent stress is a kind of measure of the distortion energy in a deformed elastic body. Note that only upper half of the mesh is depicted. The contour line values are (in unit of GPa) :
0.08
$0.19 \quad 0.32$
$0.47 \quad 0.68$
$0.87 \quad 1.13$ 
$t=4.8$
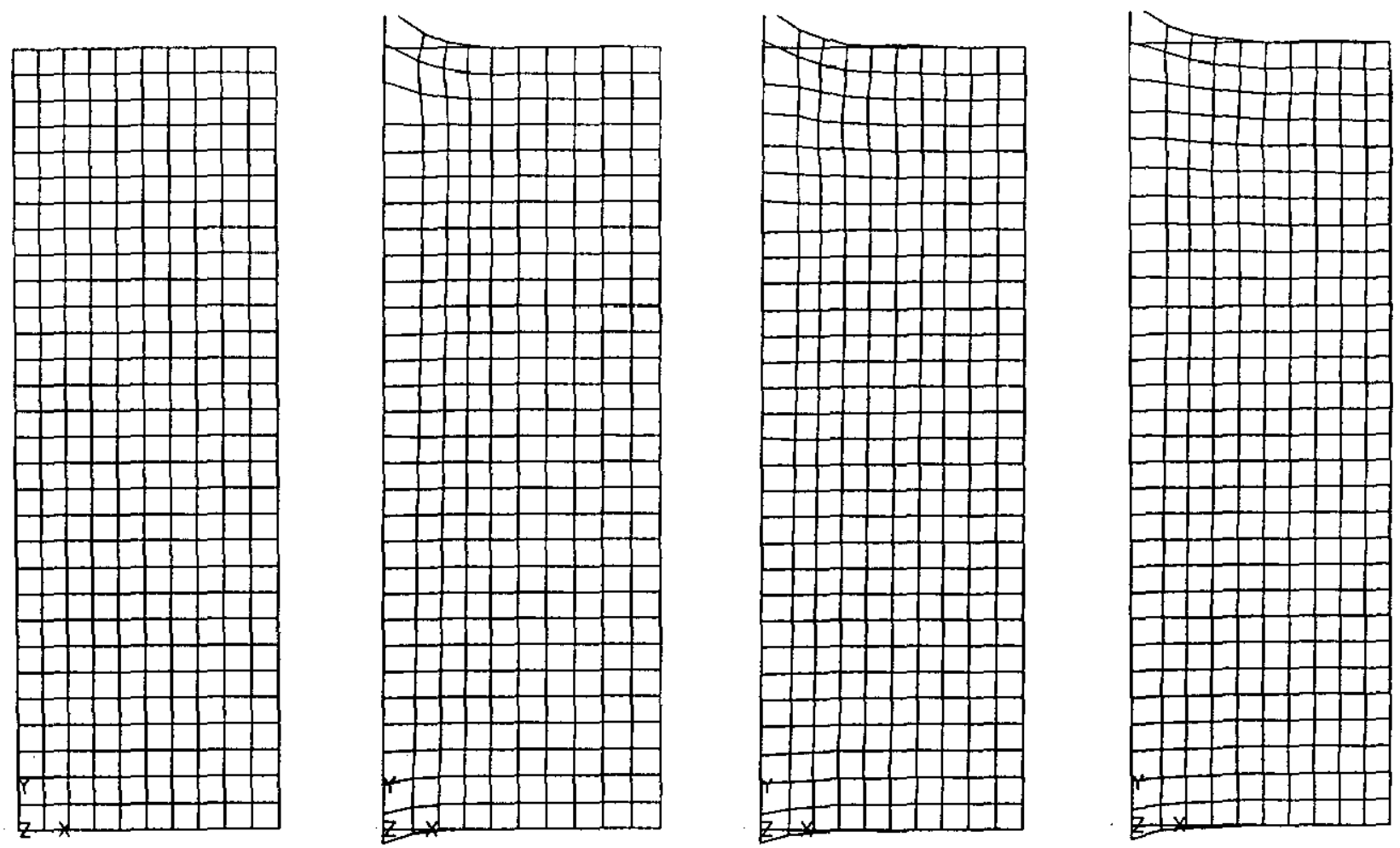

$$
t=6.4
$$

$$
t=8.0
$$

$$
t=9.6
$$

$$
t=11.2
$$
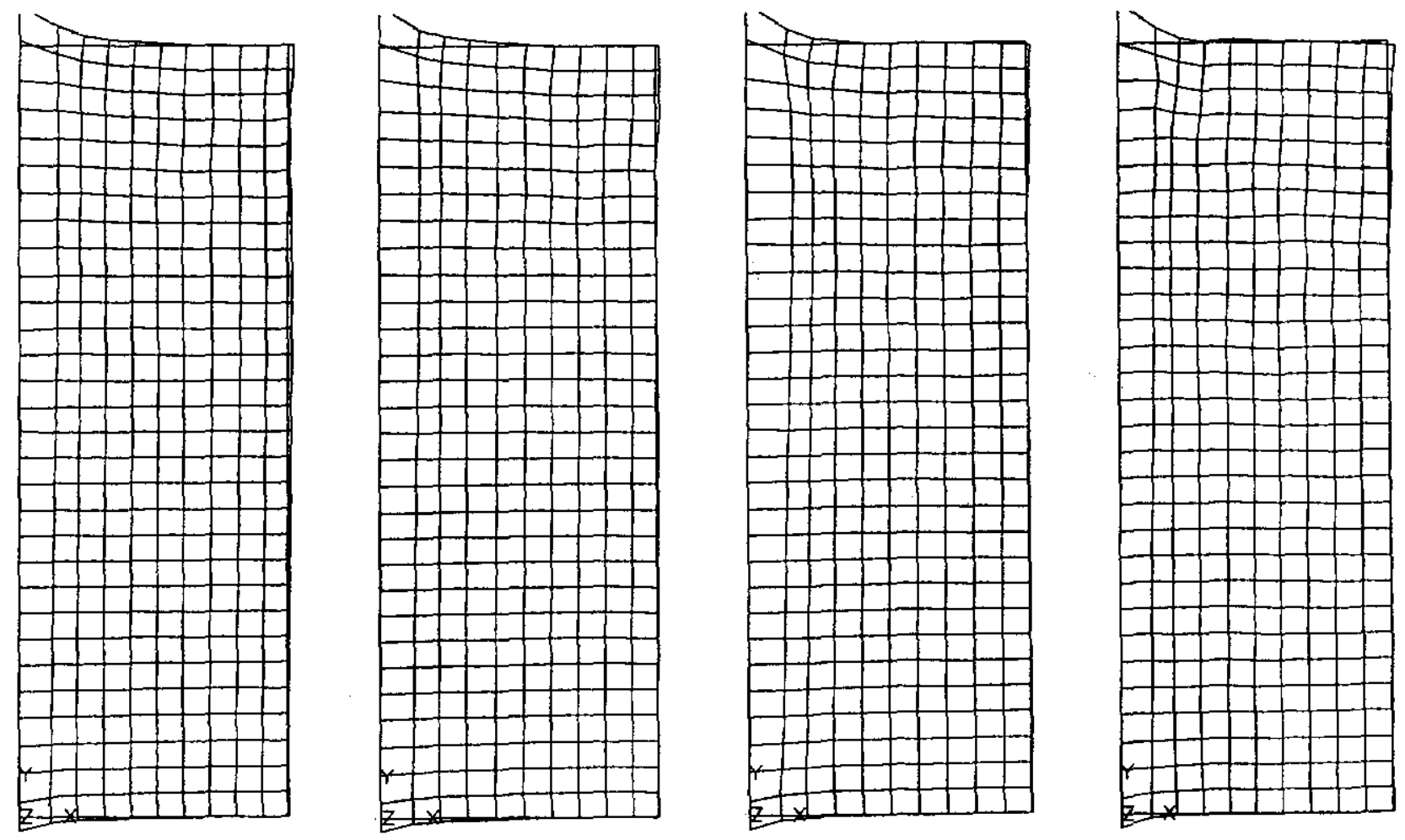

Fig. 15 Deformation of a finite length target 

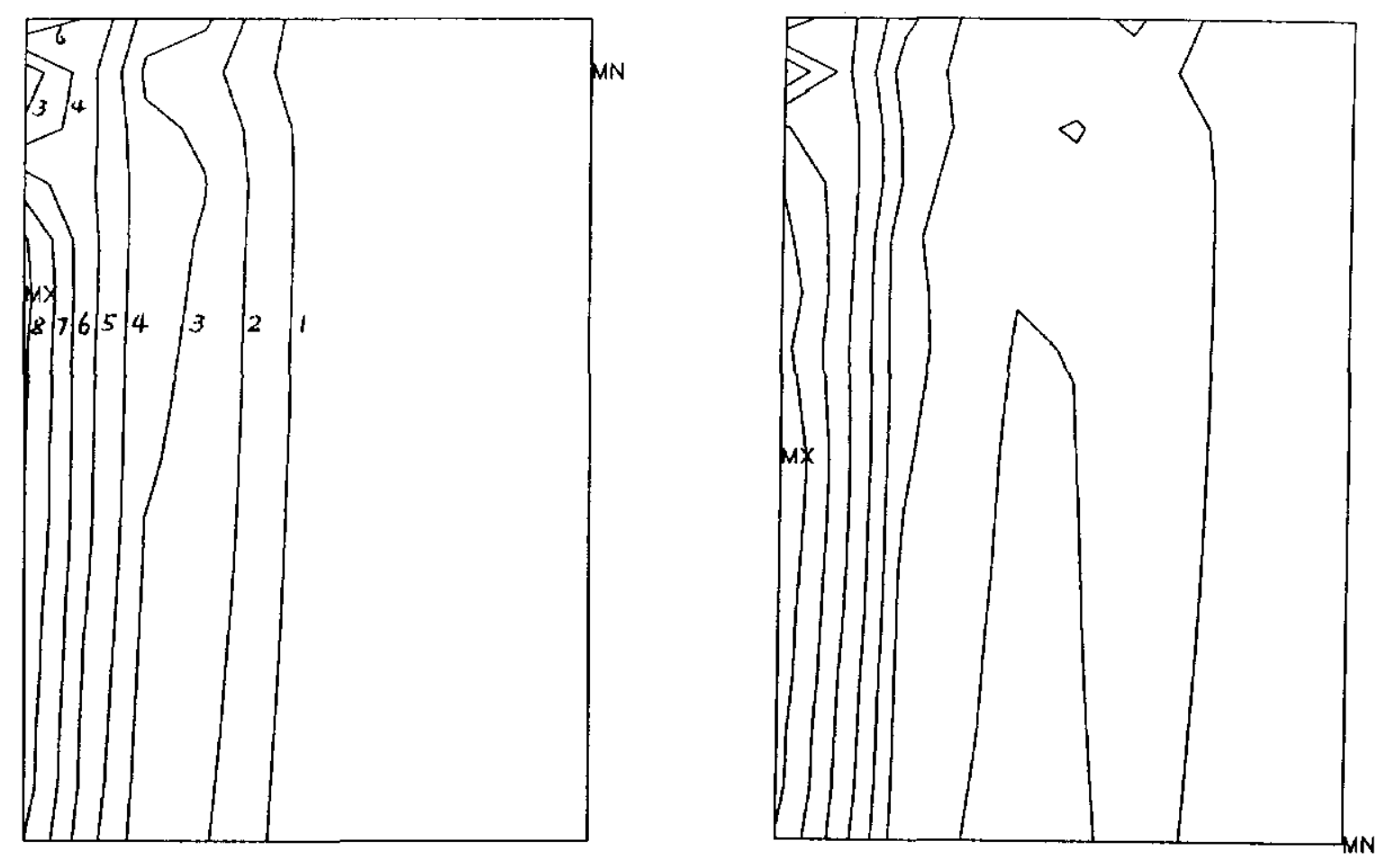

$t=1.6$

$t=3.2$

$\mathrm{t}=4.8$

$t=6.4$
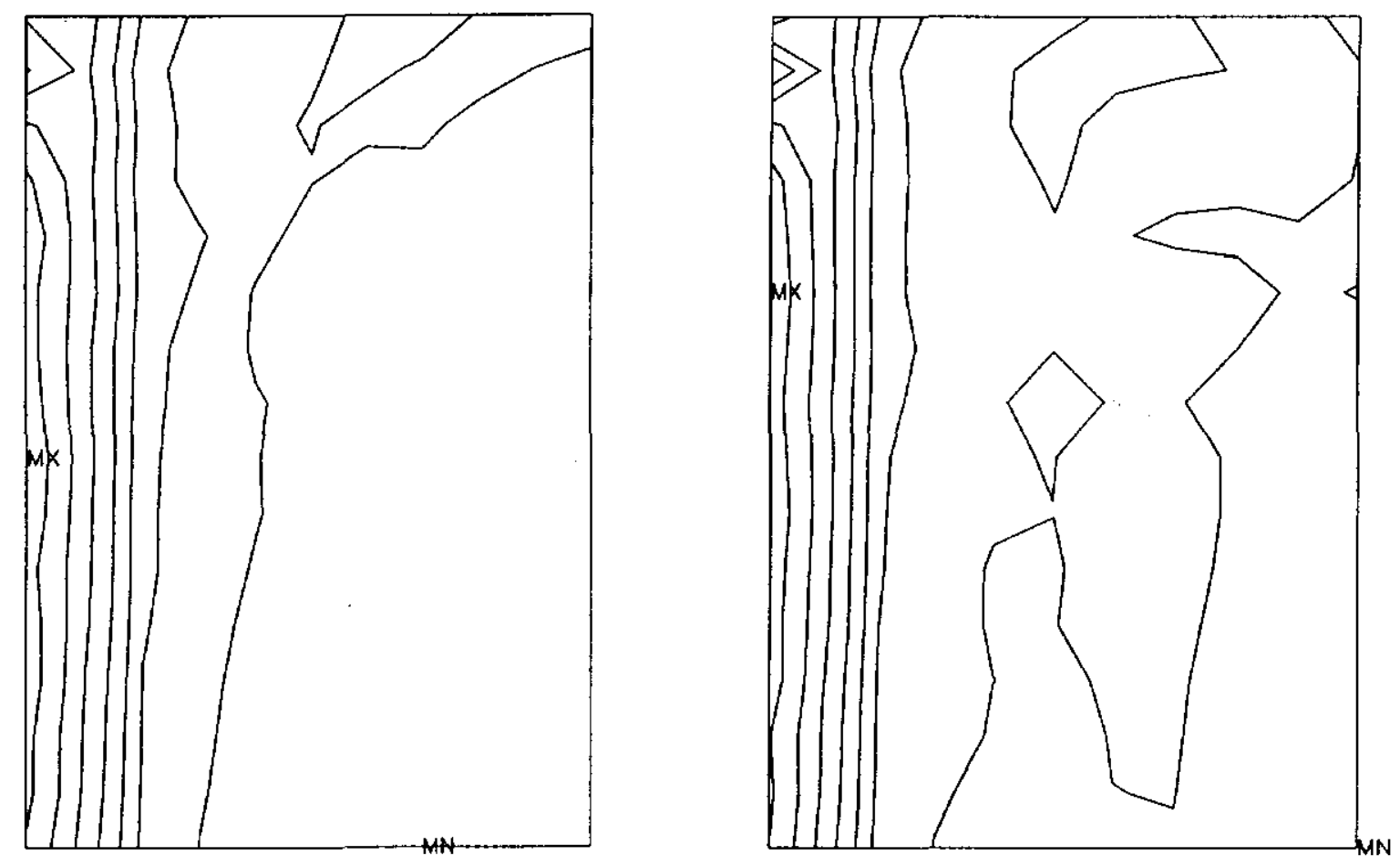

Fig. 16 Contour curves of equivalent stress 


\section{Design Considerations}

From our analysis, several sources of potential danger to the target has been identified (Fig. 17):

- At the center of target, the volume (hence density) change is maximum, this may decrease the antiproton yield;

- Near the center, the shear deformation is very large, this may cause shear band and strain softening, and decrease the strength of material;

- Still away from center, where the tensile stress attains maximum, microfractures may accumulate under repeated loadings;

- At the end of target, if there is no confinement, spillout may occur.

All these should be taken care of if we are going to increase proton beam strength and the target is expected to serve reasonable time period.

To reduce the first two of above listed effects, preload pressure may be applied. By setting core region in high pressure, thermal expansion will be reduced and the stress state will be more isotropic, results in less shear deformation. As for the end portion, to prevent the target from spillout, two alternatives can be considered. The first is by drilling a hole to the back end face of the target, using the arch effect to release the pressure. The second is to use end preloading cap to confine the deformation.

The main concern here is to reduce the tensile stress resulted from reflect shock wave. so that dynamic fracture will not happen. Two ideas are pursued in this direction. the first is to use an array of holes surrounding the core region to disperse the wave. The second is to use the concept of momentum trap to prevent reflected tensile wave from going back to the center of target; We discuss these two methods in following.

The design of a copper cylinder surrounded by an array of cooling holes seems to be perfect to disperse the shock wave. An one dimensional model has been investigated and the results suggest that one layer of holes can disperse about fifty percent of the shock energy hence significantly reduce the peek of reflected tensile wave. For our cylindrical geometry, the result is even better. Because of its simplicity and integrity, this design is the first choice of the target.

Fig. 18 is the transmission coefficient for one dimensional model, where $n$ is the ratio of cross sectional area, i.e. the area with holes subtracted. $E_{1}$ is the incident 
wave energy, $E_{3}$ is the transmitted wave energy, the reflected wave energy is therefore $E_{1}-E_{3}$. The solid line and dashed line are, respectively

$$
\frac{E_{3}}{E_{1}}=\frac{16 n^{2}}{(1+n)^{4}} \quad \text { and } \quad \frac{E_{3}^{*}}{E_{1}}=\frac{2 n}{1+n^{2}}
$$

The idea of momentum trap works like this: Instead of a whole block copper as a target, we can use several co-axial cylinders. In this case, the compressional wave can go through the interface, but the reflected tensile wave can not pass the interface, because there is no bonding at interface it cannot support tensile stress.

An ANSYS run is performed for a target with a gap and the result is compared with that of a whole target. Fig. 19 is the displacements of corresponding gap nodes, and Fig. 20 is the reaction force at the gap. Fig. 21 and 22 are radial and hoop stresses in first few elements. Compare with those without gap in Fig. 23 and 24, we note that the tensile component of reflected wave has been chopped off in Fig. 21 and 22. 


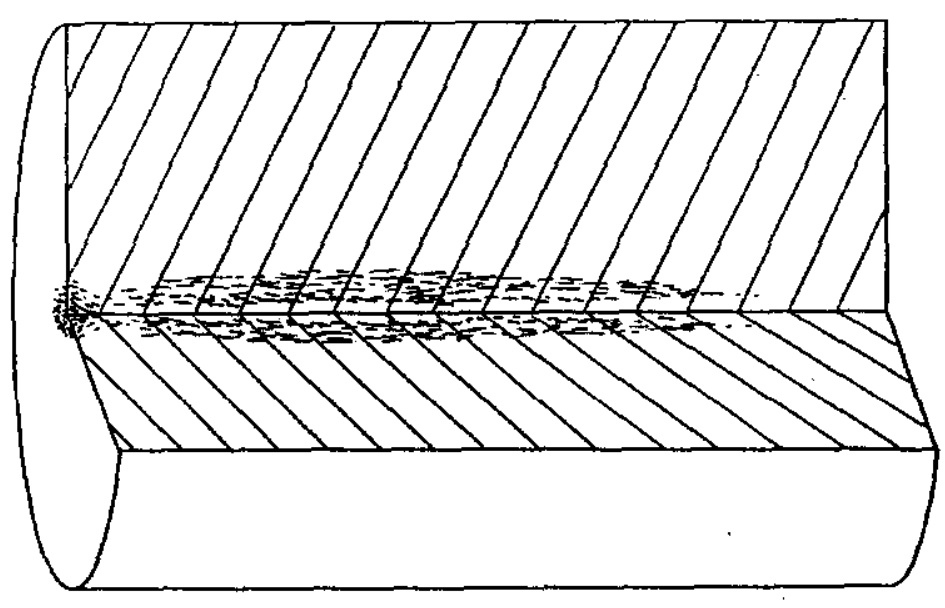

Fig. 17 potential damage of target

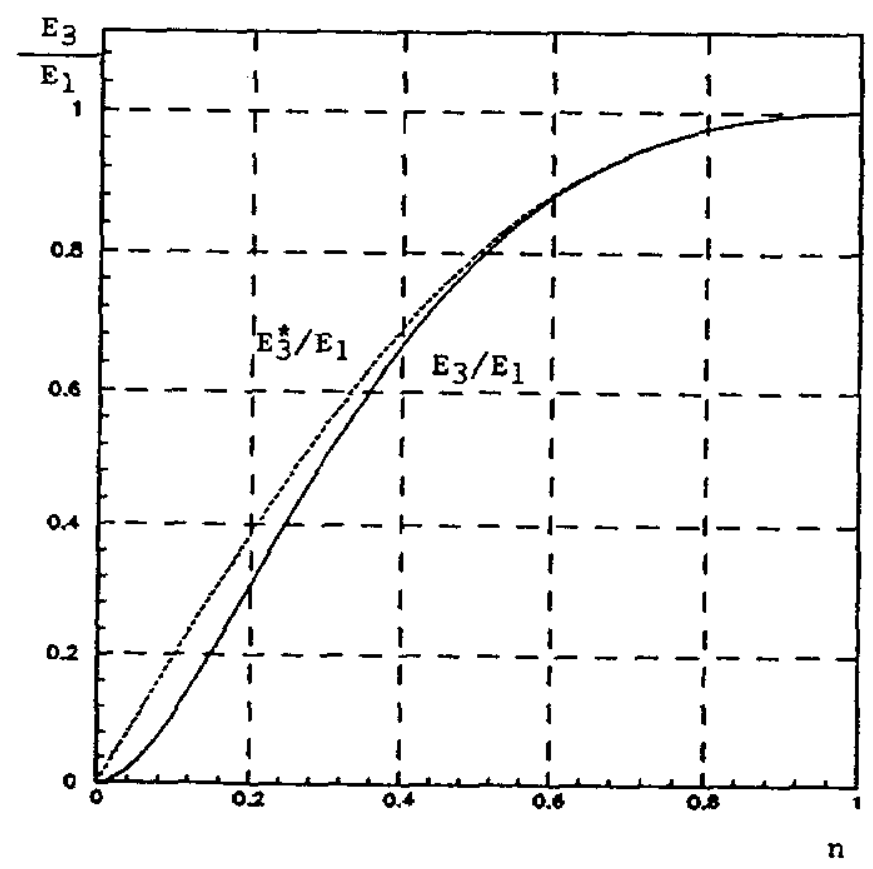

Fig. 18 Reflection coefficient of a 1-D model 


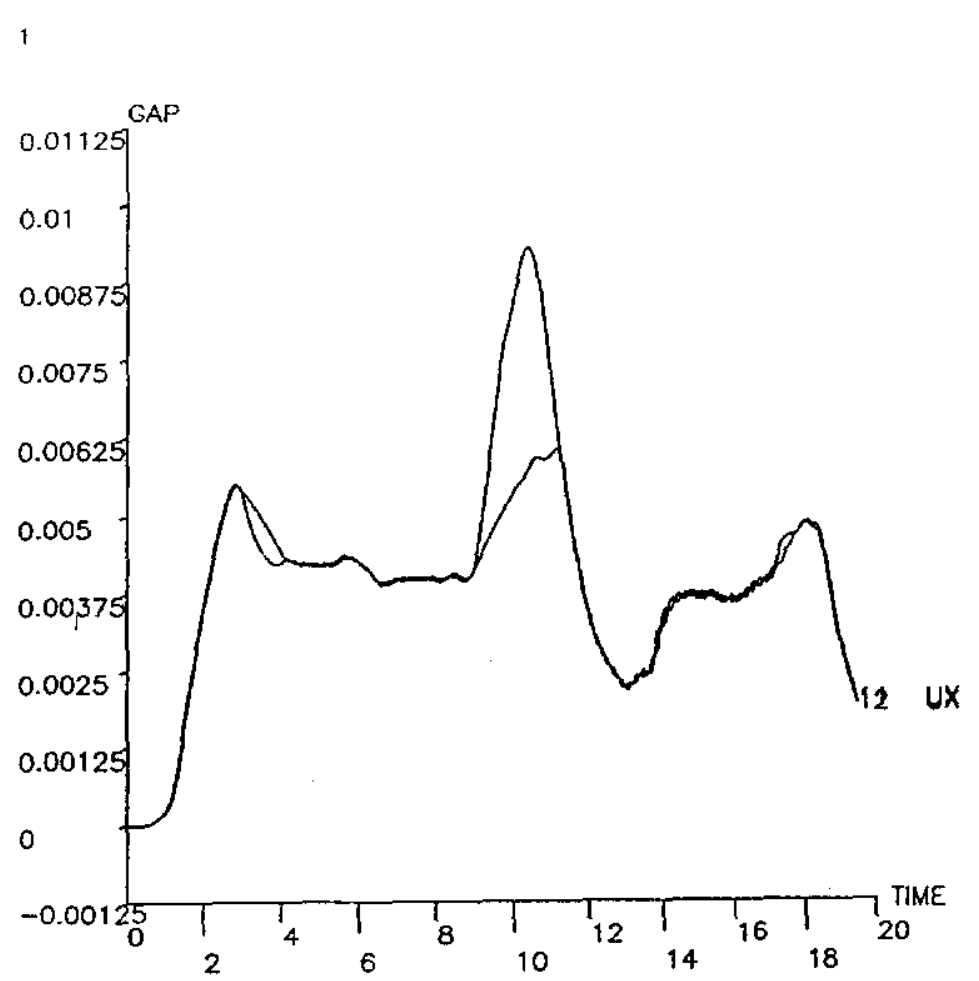

\section{Fig. 19 Gap node displacement}

Iransient thermal loeding / with oad

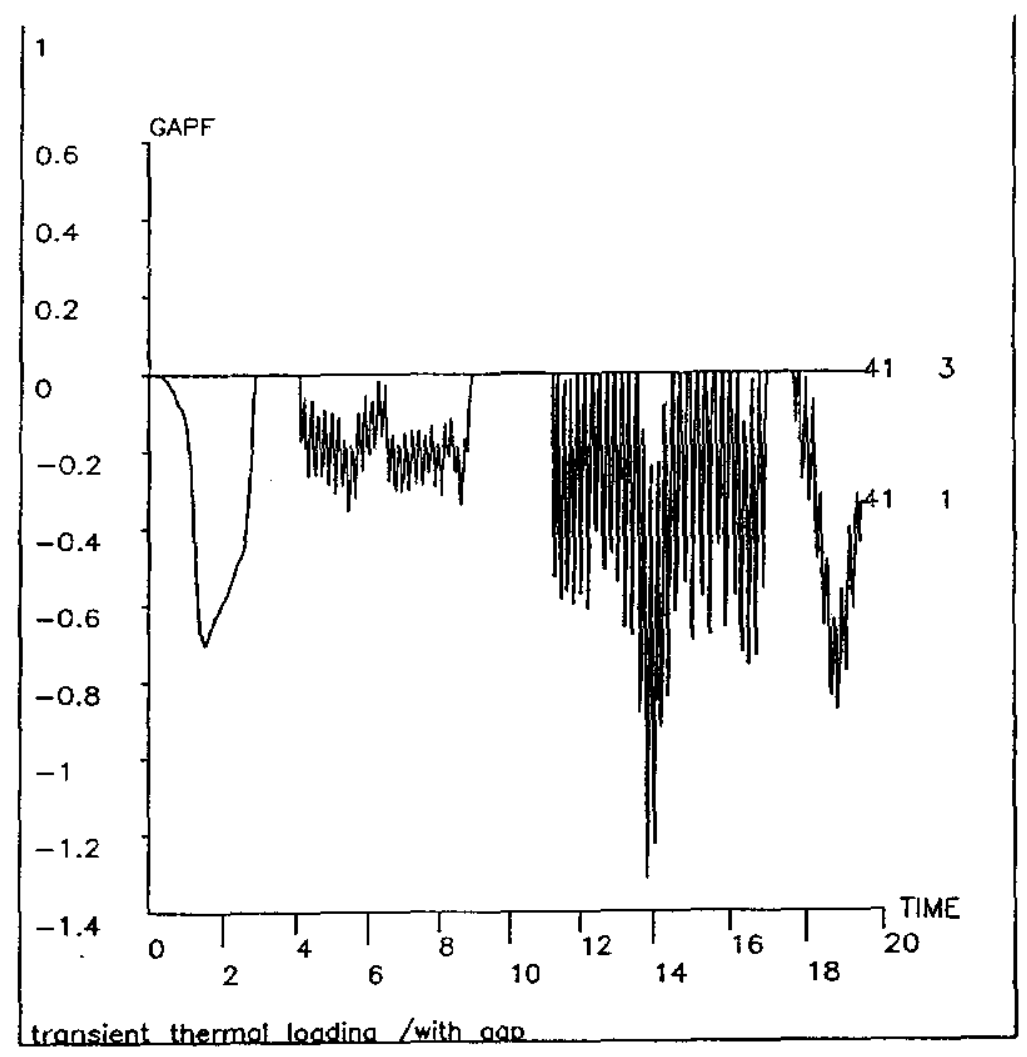

Fig. 20 Gap force 


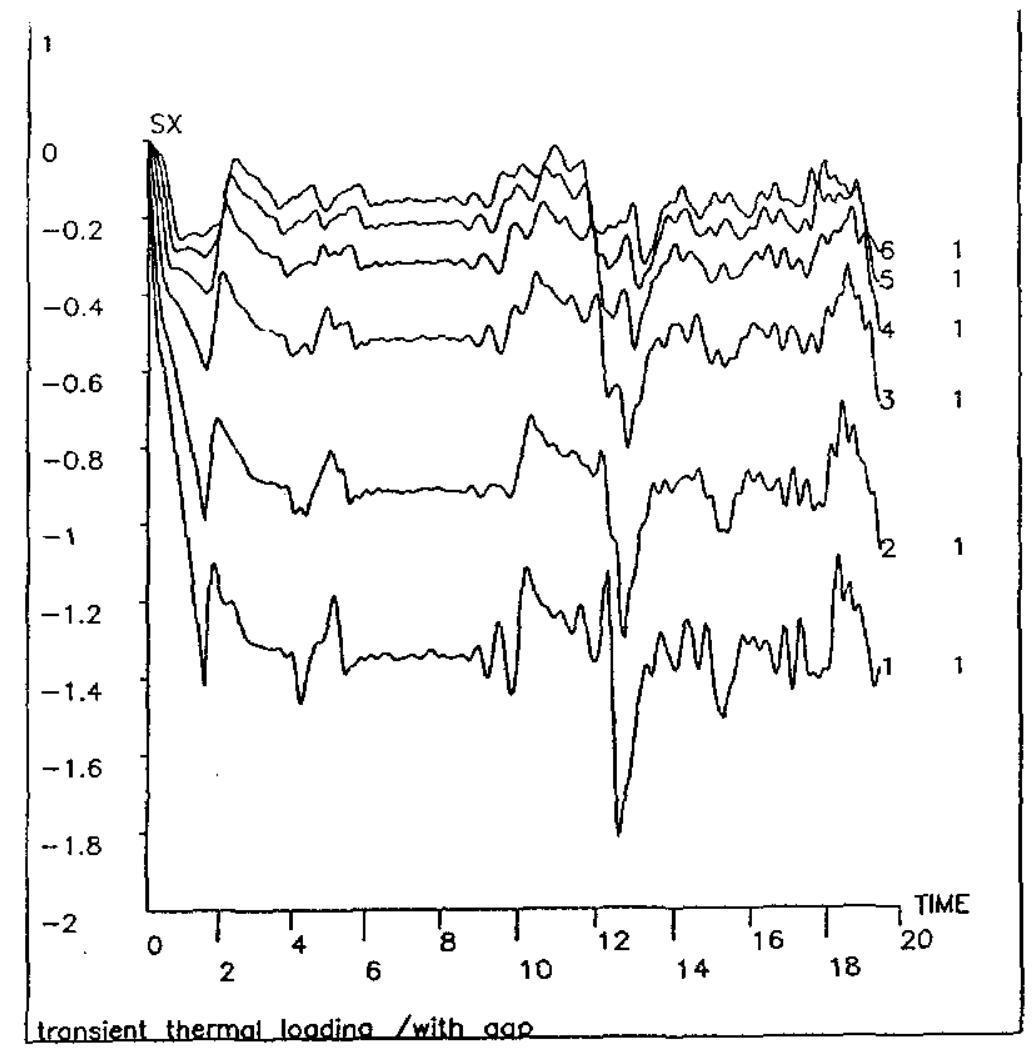

Fig. 21 radial stress

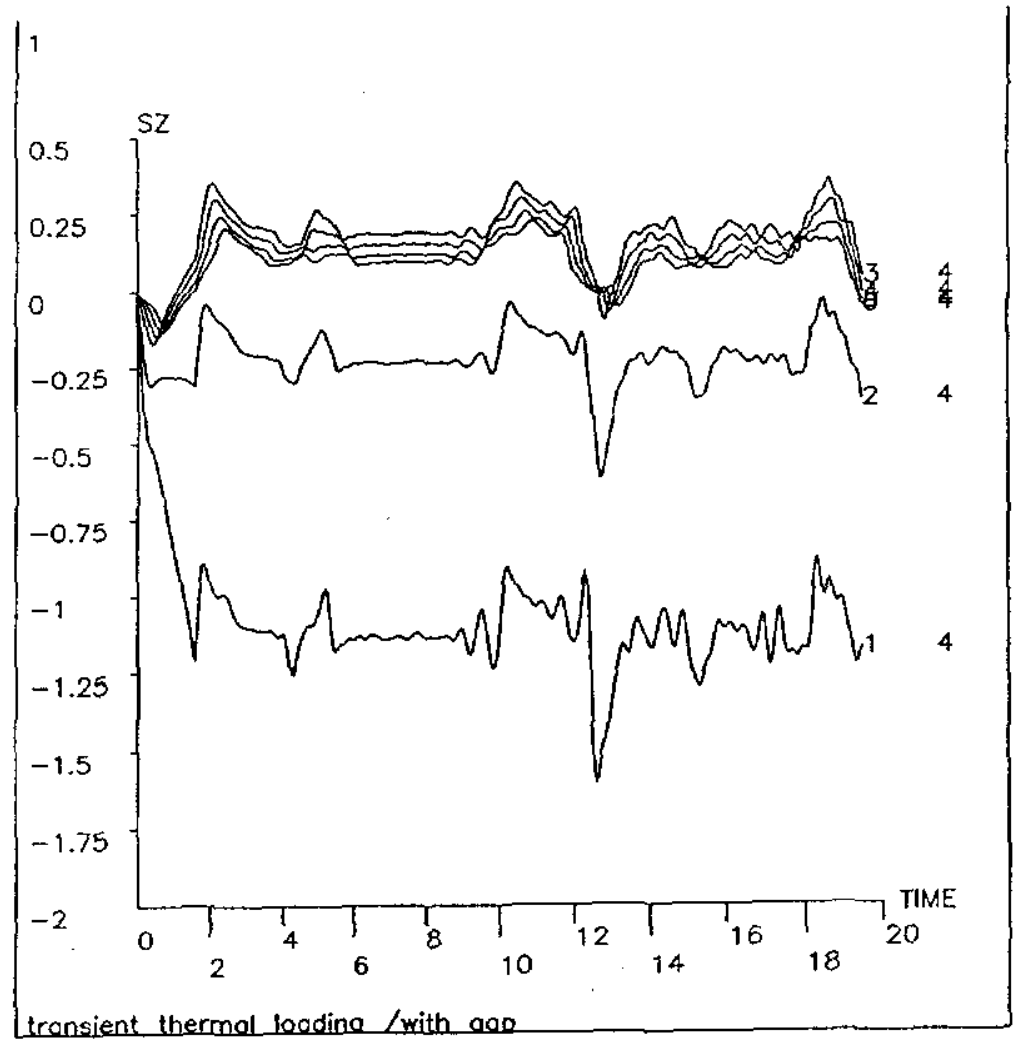

Fig. 22 Hoop stress 


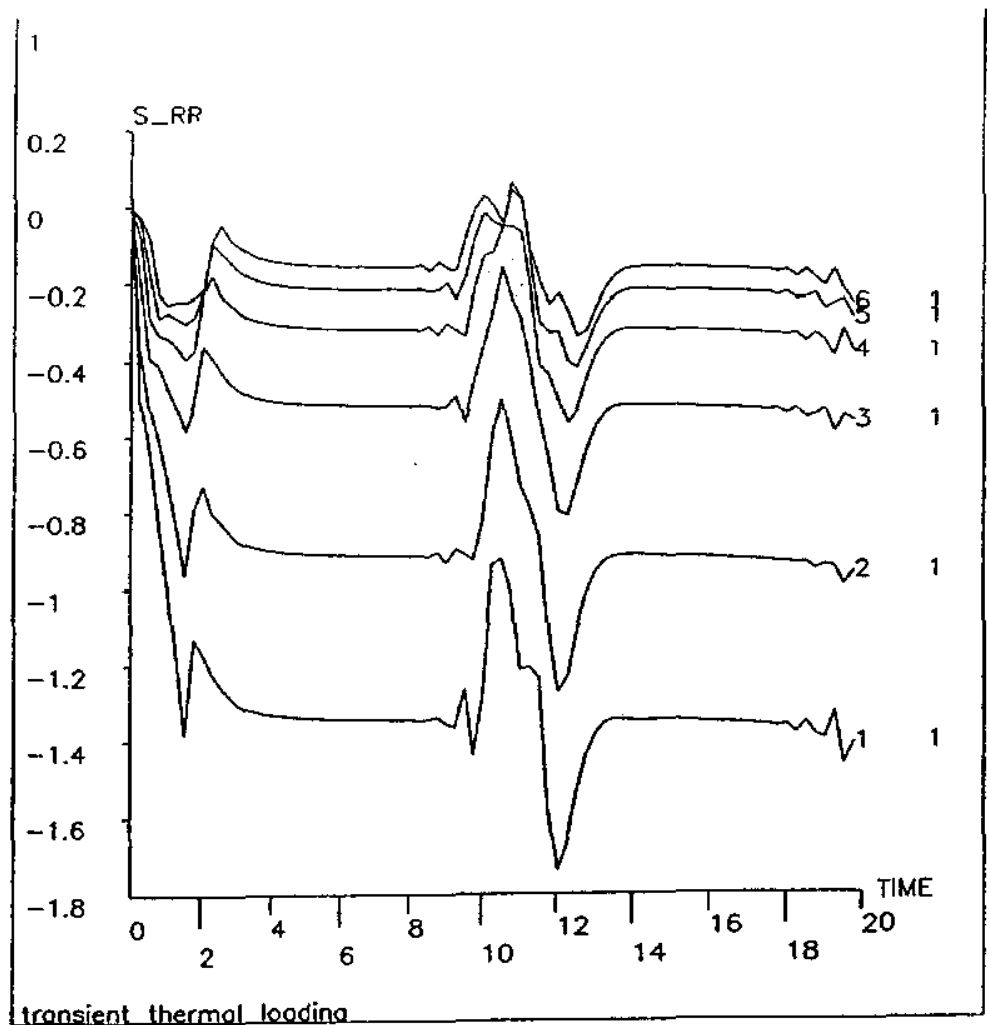

Fig. 23 Radial stress without gap

Fig. 24 Hoop stress without gap 


\section{Concluding Remarks}

A few statements have to be made before closing this report.

First, it should be noted that our finite element mesh size is $0.625 \mathrm{~mm}$ while the proton beam size is $\sigma=0.1 \mathrm{~mm}$. That means the energy deposition density is averaged in the element scale; the real maximum energy deposition well beyound that presented in section 2. If protons per pulse is $5.0 \times 10^{12}$, the maximum energy deposition density per pulse will reach 2800 joules per gram (Bhat). This will inevitably bring up some difficulties, like localized melting, etc.. The detailed pictured, both thermal and mechanical, of such a high energy density is not clear at present stage, and needs further investigation.

Second, the analysis results depend on the material properties, like modulus, yield stress and strength, which are functions of composition, heat treatment, temperature, pressure, rate of deformation, and even history of deformation. It is simply impossible to consider all these factors. In above analysis, only the dependence of material properties on temperature is included. Even though, the material data are only considered by the authors to be adequate, or typical, because the scattering of data in literature. As the energy deposition increase, material reaches melting point, it should be pointed out that the description of material across the melting point is very difficult.

The effects of radiation on structural materials, including copper, are well known, like dislocation pinning, Frenkel defects (pairs of latice vacancies and interstitial atoms). The result is the increase of material strength, both yield strength and tensile strength, and some times embrittlement of the material. In our case, the proton beam intensity is very high, the interaction of target material with incident protons and seconary particles is very strong, and we expect its effect on mechanical properties of material be quite severe. Again, the quantitative description is difficult.

Third, to further understand the shock wave phenomena, some experimental verification is needed. Presently, the exploding wire experiments are under way at Argonne National Labs. We hope it can provide a method, to verify our model used in numerical analysis, and, at least in some extend, to simulate the beam line situation of the target. Thus different design ideas can be verified and compared. 